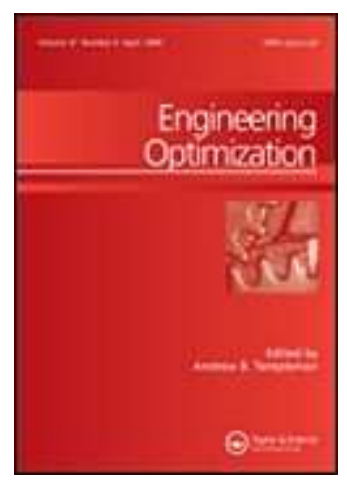

\title{
Topology Optimization for Thermal Insulation: an Application to Building Engineering
}

\begin{tabular}{|c|c|}
\hline Journal: & Engineering Optimization \\
\hline Manuscript ID: & GENO-2010-0149.R2 \\
\hline Manuscript Type: & Original Article \\
\hline $\begin{array}{r}\text { Date Submitted by the } \\
\text { Author: }\end{array}$ & 15-Dec-2010 \\
\hline Complete List of Authors: & $\begin{array}{l}\text { Bruggi, Matteo; University of Pavia, Department of Structural } \\
\text { Mechanics } \\
\text { Cinquini, Carlo; University of Pavia, Department of Structural } \\
\text { Mechanics }\end{array}$ \\
\hline Keywords: & topology optimization, thermal insulation, building engineering \\
\hline \multicolumn{2}{|c|}{$\begin{array}{l}\text { Note: The following files were submitted by the author for peer review, but cannot be converted } \\
\text { to PDF. You must view these files (e.g. movies) online. }\end{array}$} \\
\hline $\begin{array}{l}\text { engopt_bc_final2.tex } \\
\text { macrosaaa.tex } \\
\text { macrosbbb.tex } \\
\text { gENO2e.cls }\end{array}$ & \\
\hline
\end{tabular}

\section{SCHOLARONE Manuscripts}


Engineering Optimization

Vol. 00, No. 00, 00 0000, 1-19

\title{
Topology Optimization for Thermal Insulation: an Application to Building Engineering
}

\author{
Matteo Bruggi* and Carlo Cinquini \\ Department of Structural Mechanics, via Ferrata 1, University of Pavia, I27100, \\ Pavia, Italy \\ (Received 00 Month 200x; final version received 00 Month 200x)
}

\begin{abstract}
The paper deals with a numerical implementation for topology optimization that is based on the heat conduction equation and addresses problems such as the optimal design of thermal insulation in building engineering. The formulation handles heat diffusivity under the steady-state assumption for a domain with assigned convective-like boundary conditions. The optimization framework is implemented within a general-purpose finite elements code that is set to iteratively solve the thermal problem, thus allowing for a straightforward handling of two-dimensional and three-dimensional problems. A few numerical results are firstly presented to compare classical formulations for maximum heat conduction and the addressed scheme for optimal thermal insulation. The proposed methodology is therefore exploited to cope with issues peculiar to the optimal design of building envelopes, as the mitigation of the effects of thermal bridges and the design for minimum thermal transmittance of the components of a modular curtain wall.
\end{abstract}

Keywords: topology optimization; thermal insulation; building engineering;

\footnotetext{
*Corresponding author. Email: matteo.bruggi@unipv.it
} 


\section{Introduction and motivation}

Topology optimization is a powerful design tool that may be exploited to support the design process of several engineering systems. The standard formulation addresses the issue of finding the distribution of isotropic material that minimizes an objective function for an assigned set of constraints. This mathematical framework was originally exploited to cope with mechanical problems, see i.e. the pioneering work of Bendsøe and Kikuchi (1988), and, since then, it has been successfully extended to a wide range of applications. Reference is made, among the others, to Bendsøe and Sigmund (2003) and to Eschenauer and Olhoff (2001) for comprehensive reviews on the advances of the discipline, concerning both theoretical and practical issues.

A fertile area of research involves thermal problems. The case of heat conduction has been extensively investigated in fundamental works, see e.g. Cherkaev (2000) and Allaire (2002), having the main aim of providing a sound mathematical insight to many continuous settings arising in topology design. Referring to the applications, most of the relevant formulations address the problem of the optimal conductor, i.e. the achievement of the topology that maximizes heat transfer for an assigned volume constraint under steady-state assumption. This is the case of the work in Li et al. (1999) that presents an implementation of the evolutionary structural optimization (ESO) framework to conduction-dominated thermal applications. The same optimization technique is used to address temperature reduction of heat conducting fields in Li et al. (2004). The constitutive equations peculiar to heat problems have been handled not only within the classical scheme of finite elements discretization, but also resorting to the finite volume method, as proposed in Gersborg-Hansen et al. (2006). Other formulations refer to the adoption of level set methodologies to solve the relevant minimization problem, see e.g. Zhuang et al. (2007). The interest towards the achievement of optimal conductors has driven significant advances in specific application where thermal loads and boundary conditions may not be considered as design-independent. Reference is made e.g. to Gao et al. (2008), that addresses load effects in conduction-dominated problems with heat generation depending on the material state, or to Bruns (2007) and Iga et al. (2009), that propose ad hoc methodologies to handle the effects of evolving structural boundaries also in the case of convection-driven problems. Thermal aspects are taken into account within several multi-physics formulations, as in the case of the optimal design of thermo-elastic components addressed in Cho and Choi (2005), Habbal et al. (2004) and Diaz and Benard (2003). Peculiar issues of the optimization for the heat conduction problem are discussed in the multi-material design presented in Zhuang et al. (2010) and in the nano-scale investigations of Evgrafov et al. (2008).

As outlined above, most of the literature referring to heat problems has widely investigated the topology design for maximum conductivity, while thermal insulation has not received as much attention. Fundamentals of the problem may be directly derived e.g. in Donoso and Sigmund (2004). The authors address topology optimization for multiple physics problems that are modeled by the Poisson's equation, dealing with numerical schemes that are able to minimize or maximize suitable objective functions also in the case of heat transfer. Recent trends of civil and mechanical engineering show an increasing interest towards the themes of sustainable design and energy saving, especially in the area of building technology. On this theme, the work in Munoz et al. (2007) extends to the field of topology optimization the problem that was originally discussed in Buttazzo (1988), i.e. the optimal design of thin insulating layers around conductive media. The authors aim to find the best distribution of a fixed amount of insulation to 
be located around a non-design conductive domain that is heated by an internal source. They formulate the problem both in the format of a topology optimization design, i.e. distributing a fraction of insulating material, and adopting a shape design scheme, i.e. distributing a thin layer of insulation. Both formulations may be actually seen as a preliminary approach for the achievement of the optimal layout of insulation around a fixed indoor environment with assigned radiators.

This contribution addresses the topology optimization for thermal insulation adopting a different perspective, i.e. dealing with the maximization of the performances of the building envelope for energy saving. Based on the above literature on heat transfer, an algorithm is especially conceived and implemented to minimize the thermal transmittance of elements such as curtain walls, floors or roof systems and to reduce the effects of undesired thermal bridges. The proposed procedure exploits the steady-state heat equation for conduction-dominated problems with convection-like boundary conditions and resort to the adoption of a general-purpose finite elements code along with mathematical programming, for the solution of the minimization setting. The optimal distribution of the isotropic material is managed through a RAMP-like scheme, see Stolpe and Svanberg (2001), that interpolates thermal conductivity depending on the material density. The finite elements subroutines solve the heat equations to iteratively provide the objective function and the relevant sensitivities to the optimization algorithm, herein the Method of Moving Asymptotes (MMA), see Svanberg (1987). A simple procedure is used to update the thermal properties within the environment of the general-purpose code, as similarly done in Bruggi (2009) for the minimization of structural compliance. This allows to tackle in a straightforward way both the two-dimensional problems, that are usually addressed in most of the current literature, and the three-dimensional simulations.

The outline of the paper is as follows. Section 2 reports fundamentals of steady-state equations for the heat conduction problem, defining the framework herein investigated. Section 3 presents the optimization scheme, paying peculiar attention both to the formulation and to its implementation within the adopted general purpose finite elements code. Section 4 firstly discusses the difference between the results achieved through classical optimization procedures for maximum heat conduction and the adopted optimization for thermal insulation. The implemented numerical procedure is therefore tested on applications that concern the preliminary design for minimum transmittance of the components of a building envelope.

\section{Governing equations}

This section reports fundamentals on the continuous form of the steady-state heat conduction problem as it will be addressed in the sequel. A general treatment of the heat transfer equations may be found in the literature mentioned above and is herein specialized to the case where a suitable set of convective-like boundary conditions drive the problem.

Let $\Omega \in R^{2}$ define the domain, $\partial \Omega$ its regular boundary and $\underline{\underline{k}}$ the second order thermal conductivity tensor in the case of a linear isotropic material with thermal conductivity $k$. Let $T$ denote the unknown temperature field. One may generally enforce an assigned temperature distribution $T_{t}$, i.e. $T=T_{t}$ on $\Gamma_{t}$, or a prescribed heat flux $q$, i.e. $-(\underline{\underline{k}} \operatorname{grad} T) \cdot \underline{n}=q$ on $\Gamma_{f}, \underline{n}$ being the normal to the relevant boundary $\Gamma_{f}$. A convective heat transfer on the surface $\Gamma_{c}$ may be also considered, as a special case of the latter 
condition. The heat flux per unit area $q_{c}$ is proportional to the difference in temperature between the surface of the body and the ambient flow $T_{a}$, i.e.:

$$
q_{c}=h_{c}\left(T-T_{a}\right)
$$

being $h_{c}$ the so-called convective heat transfer coefficient. The above formula is commonly used in the design of building envelopes where the constant $h_{c}$ globally takes into account the heat fluxes interesting the body surfaces under laminar flow condition, see e.g. ISO 6946:2007.

The building envelope is herein modeled as a conduction-dominated solid domain that exchanges heat fluxes with fluid regions along its boundaries. The heat equation is therefore governed by an assigned set of convective boundary enforcements that take into account indoor and outdoor conditions on $\Gamma_{c i} \cup \Gamma_{c o}=\Gamma_{c}$, through a prescribed distribution of ambient temperatures and convective coefficients. No heat source, heat flux $q \neq 0$ or fixed value of the temperature field is additionally provided within the domain or at the boundary. A region $\Gamma_{f}$ may be considered to enforce a null heat flux along a prescribed zone of $\partial \Omega$, due e.g. to symmetry conditions.

According to the above assumption, let address the case of an outdoor ambient temperature $T_{a o}=0$, while an indoor one $T_{a i}>0$. Following e.g. Gosz (2006), the consequent variational formulation for the considered heat transfer problem reads: find $T \in \mathcal{V}=\left[H^{1}(\Omega)\right]^{2}$ such that $\left.T\right|_{\Gamma_{t}}=T_{t}$ and

$$
\int_{\Omega} \operatorname{grad} w \cdot(\underline{\underline{k}} \operatorname{grad} T) d x=-\int_{\Gamma_{c i}} w h_{c}\left(T-T_{a i}\right) d s-\int_{\Gamma_{c 0}} w h_{c} T d s, \quad \forall w \in \mathcal{V}
$$

In view of the adoption of a suitable objective function for the optimization problem it is useful to refer to the well-known thermal compliance $\mathcal{C}$. This may be derived from the heat equation in full analogy with the definition of structural compliance for the elasticity problem, see e.g. Cherkaev (2000) and Allaire (2002). Within the considered case, the thermal compliance $\mathcal{C}(T)$ reads:

$$
\mathcal{C}(T)=<T, h_{c} T_{a i}>_{\Gamma_{c i}}=a(T, T)+<T, h_{c} T>_{\Gamma_{c}},
$$

where the relevant bilinear forms refer to a straightforward manipulation of Eqn. (2). For a given distribution of convection coefficient $h_{c}$ and an assigned set of ambient temperature $T_{a o}=0$ and $T_{a i}>0$, the heat diffusion depends on the magnitude of the convective flux crossing $\Gamma_{c i}$, i.e. $h_{c}\left(T_{a i}-T\right)$. Looking at Eqn. (3) one easily concludes that minimization of $\mathcal{C}$ solves the problem of the optimal thermal conductor, meaning that the flux $h_{c}\left(T_{a i}-T\right)$ is maximized due to the minimization of the relevant surface temperature $T$ on $\Gamma_{c i}$. Maximization of $\mathcal{C}$ conversely addresses the case of the optimal thermal insulator, where minimum heat conduction is aimed. It must be remarked that the compliance $\mathcal{C}$ may also be computed in terms of the bilinear forms $a(T, T)$ and $<T, h_{c} T>_{\Gamma_{c}}$, as exploited in the implementation presented next.

Reference is made e.g. to Donoso and Sigmund (2004), Bruns (2007), Gao et al. (2008) and Iga et al. (2009) for a discussion on the choice of suitable objective functions for the maximization of heat conduction in several classes of engineering applications. Eqn. (3) is actually a subset of the available energy-based functionals that is well-suited to cope with the herein considered thermal insulation problem. 


\section{Optimal design for thermal insulation}

The previous section has shown fundamentals of the relevant heat conduction problem, towards the application of optimization techniques to the engineering of the building envelope. The sequel focuses on the key elements of the proposed optimization scheme, referring to material interpolation, optimal formulations and details on the implemented procedure.

\subsection{Thermal conductivity interpolation model}

Topology optimization by distribution of isotropic material is based on the adoption of a suitable interpolation scheme, see e.g. Bendsøe and Sigmund (2003), Zhou and Rozvany (1993), to approximate the constitutive properties of the material, herein the thermal conductivity $k$, depending on the unknown density field $\rho$. The proposed procedure implements a so-called RAMP model, i.e. a Rational Approximation of Material Properties Stolpe and Svanberg (2001), that reads:

$$
k(\rho)=k_{\rho=0}+\frac{\rho}{1+p(1-\rho)}\left(k_{\rho=1}-k_{\rho=0}\right) .
$$

In the above equation the parameter $p \geq 0$ is used to interpolate between two extremal values of the conductivity, $k_{\rho=0}$ and $k_{\rho=1}$. The optimal procedure aims in fact to distribute two material phases, i.e. $\rho=0$ and $\rho=1$. The first refers to the less conductive fraction, while the second defines the more conductive phase, meaning that $k_{\rho=1}>k_{\rho=0}$. For $p=0$ the above RAMP model provides a linear interpolation between $k_{\rho=0}$ and $k_{\rho=1}$, while larger values of the governing parameter produce a strong penalization of the intermediate range and encourage the achievement of the expected pure $0-1$ designs. As found in the numerical section, the assumption $p=3$ allows to successfully address the considered two-phase optimizations, meaning that pure $0-1$ layouts are achieved without the appearance of any grey region.

\subsection{Problem formulation}

All the studies devoted to solve the problem of the optimal thermal conductor on a domain $\Omega$ with assigned convective boundary conditions may be framed within the following setting:

$$
\left\{\begin{aligned}
\text { s.t. } & \left.\int_{\Omega} \operatorname{grad} w \cdot \underline{\underline{k}}(\rho) \operatorname{grad} T\right) d x=-\int_{\Gamma_{c}} w h_{c}\left(T-T_{a}\right) d s \forall w \in \mathcal{V} \\
& \int_{\Omega} \rho d x \leq V_{f} V_{t o t} \\
& 0 \leq \rho \leq \bar{\rho}=1
\end{aligned}\right.
$$

In the above statement, the objective function is the bilinear form of Eqn. (3), whose temperature $T$ fulfills Eqn. $(5)_{2}$, i.e. the state condition for the considered heat conduction problem. The same equation points out the dependence of the thermal conduction tensor $\underline{\underline{k}}$ on the unknown design variable $\rho$, according to Eqn. (4). In agreement with standard assumptions peculiar to the applications herein investigated, the coefficients $h_{c}$ 
are referred to fixed boundaries $\Gamma_{c}$ and are independent on $\rho . V_{f}$ is a fixed fraction of the total design domain $V_{t o t}=\int_{\Omega} d x$ and refers to the admissible volume of the more conductive fraction for the two-phase design, see Section 3.1.

Variations of the above statement have been investigated by means of different objective functions and material interpolation schemes in some of the literature previously referenced. According to the aim of the paper, Eqn. (5) is herein exploited to make comparisons on numerical and applicative features with respect to the proposed formulation for the achievement of optimal thermal insulation, as presented next.

Remaining in the framework of the optimization of a domain $\Omega$ with assigned convective boundary conditions, the setting for the optimal design of thermal insulators may be written as:

$$
\left\{\begin{aligned}
& \max _{\rho \in \mathcal{R}_{0}^{+}} \mathcal{C}=a(T, T)+<T, h_{c} T>_{\Gamma_{c}} \\
\text { s.t. } & \int_{\Omega} \operatorname{grad} w \cdot(\underline{\underline{k}}(\rho) \operatorname{grad} T) d x=-\int_{\Gamma_{c}} w h_{c}\left(T-T_{a}\right) d s \forall w \in \mathcal{V} \\
& \int_{\Omega}(1-\rho) d x \leq V_{f} V_{t o t} \\
& 0 \leq \rho \leq \bar{\rho}=1
\end{aligned}\right.
$$

As detailed in Section 2, the maximization of the thermal compliance $\mathcal{C}$ allows for a straightforward minimization of the heat diffusivity through the domain $\Omega$. A minor modification is also required on the volume constraint of Eqn. $(6)_{3}$, with respect to Eqn. $(5)_{3}$. Referring to a two-phase design, the goal of the optimization is the achievement of an optimal distribution of the assigned fraction $V_{f}$ of insulating material along with the complemental amount $1-V_{f}$ of conductive medium. According to the herein adopted form of the interpolation scheme, one has to take into account that $k_{\rho=0}<k_{\rho=1}$, meaning that $\rho=0$ refers to the insulating fraction and $\rho=1$ denotes the conductive phase. Within such an assumption Eqn. (6) 3 has the suitable form that enforces an upper bound $V_{f}$ on the less conductive material fraction.

As already mentioned, an interesting approach for the topology design of optimal insulation has been considered in the work by Munoz et al. (2007). The authors move from the "thin insulation" case introduced by Buttazzo (1988) and adopt an alternative objective function with respect to Eqn. (3), mainly due to a different physical modeling of the problem. Reference is made to this contribution, along with the work in Donoso and Sigmund (2004), for a discussion on the properties of the arising max setting with respect to the classical min problem.

\subsection{Discretization}

The adopted strategy for the numerical solution of Eqns. (5) and (6) resorts to the discretization of the density field, to cope with the minimization problem via mathematical programming, and to the approximation of the temperature field, to tackle the heat state equation via the finite elements method. The introduction of the classical scheme of one density unknown for each element of the mesh is herein adopted, see e.g. Bendsøe and Sigmund (2003), Sigmund (2001b), along with quadrangular or hexahedral elements having bilinear or trilinear shape functions for temperature.

The discrete form of the thermal compliance $\mathcal{C}$ in Eqns. (5) and (6) may be therefore 
written as:

$$
\mathcal{C}=\underline{\theta}^{T}(\underline{\underline{K}}(\underline{x})+\underline{\underline{H}}) \underline{\theta}=\sum_{i=1}^{N} \underline{\theta}_{i}^{T}\left(k\left(x_{i}\right) \underline{\underline{K}}_{0, i}+\underline{\underline{H}}_{i}\right) \underline{\theta}_{i}
$$

where $\underline{x}$ is the vector of the density unknowns, being $x_{i}$ the value associated to the $i$-th of the $\bar{N}$ elements that make the mesh, and $\underline{\theta}_{i}$ denotes the elemental subset of the vector of the nodal temperatures, i.e. $\underline{\theta}$. The objective function is computed as a sum of the $N$ contributions due to vectors $\underline{\theta}_{i}$, elemental conductivity matrices $\underline{\underline{K}}_{i}$ and elemental heat transfer matrices $\underline{\underline{H}}_{i}$. Each elemental conductivity matrix may be defined as $k\left(x_{i}\right) \underline{\underline{K}}_{0, i}$, where $\underline{\underline{K}}_{0, i}$ refers to a unitary thermal coefficient. No dependence on $x_{i}$ is considered in the convective matrices $\underline{\underline{H}}_{i}$, as detailed in Section 3.2.

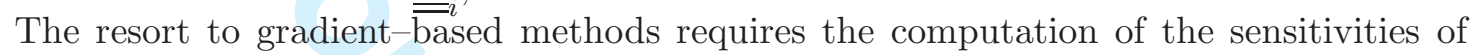
the discrete thermal compliance in Eqn. (7). One may refer to the relevant literature introduced in Section 1, also exploiting the above mentioned property of $\underline{\underline{H}}_{i}$. According to the implemented discretization of the density field, the derivative of $\mathcal{C}$ with respect to the $j$-th design variable $x_{j}$ may be directly computed as:

$$
\frac{\partial \mathcal{C}}{\partial x_{j}}=-\underline{\theta}_{j}^{T} \frac{\partial k\left(x_{j}\right)}{\partial x_{j}} \underline{\underline{K}}_{0, j} \underline{\theta}_{j}
$$

It must be remarked that the adoption of a low order finite elements discretization along with an element-based density interpolation may be affected by the arising of numerical instabilities, see e.g. Sigmund and Petersson (1998). Undesired patterns and mesh dependence may also be faced in the case of thermal insulation problems, as outlined in Munoz et al. (2007). To avoid undesired layouts and to introduce a length-scale control against mesh dependence, a filtering of the sensitivities of the objective function is classically operated before passing the gradient information to the minimizer, see e.g. Bendsøe and Sigmund (2003).

\subsection{Implementation}

As already mentioned, the discrete forms arising from Eqns. (5) and (6) are well-suited to an efficient numerical implementation, due to some similarities with respect to classical minimum compliance problems. A general-purpose finite elements software is herein adopted and linked to a main Fortran code through an application programming interface. The main code manages the FEM calls in order to compute the objective function and its sensitivities, as iteratively required by the minimization algorithm. A similar procedure has been implemented in Bruggi (2009) to address an application of the optimal design for maximum structural stiffness in two and three dimensions. Reference is also made to Sigmund (2001b) for a compact Matlab implementation of the minimum compliance problem under plane state assumptions.

The main steps of the herein proposed numerical scheme may be summarized as follows.

- Firstly the FEM software environment is exploited to define the domain and the boundary conditions. This step also includes finite elements discretization, that is herein limited to the definition of a mesh of four-node or eight-node elements, depending on the physical dimension of the problem. 
- The second step concerns the automatic generation of a different property type for each element of the mesh. This trick allows to assign the value of the conduction coefficient $k_{i}$ of the $i$-th element, depending on its current density $x_{i}$ through Eqn. (4). This procedure does not call for any manipulation on the "stiffness" matrices, which would not be allowed by the programming interface of the adopted finite elements code. At the first iteration a starting point for the minimization must be assigned. The choice $x_{i}=V_{f}$ is made $\forall i$ in the case of the optimal design of the thermal conductor, while $x_{i}=\left(1-V_{f}\right)$ is assigned if the optimal insulation problem is considered.

- This step is the core of the procedure and is fully driven by the minimization algorithm that iteratively asks for the computation of objective function, constraints and their sensitivities, to perform the topology optimization.

At each iteration a single solution of the heat equation is computed by the finite elements code in order to derive the $N$ elemental temperature vectors $\underline{\theta}_{i}$ used in Eqn. (7) and Eqn. (8) to compute the required quantities. The element conductive matrices $\underline{\underline{K}}_{0, i}$ and heat transfer matrices $\underline{\underline{H}}_{i}$ may be found by direct integration of the involved shape functions, see i.e. API Manual (2005). MMA processes the current information and provides an updated set of densities. The conduction coefficients descending from Eqn. (4) are therefore assigned to the relevant element property types that have been introduced in the previous step.

The above procedure is repeated until convergence, meaning that the difference between the values of each unknown in two subsequent iterations is less than a fixed tolerance, herein equal to $10^{-3}$.

\section{Numerical investigations}

Section 4.1 has the aim of validating the introduced numerical setting, discussing the differences between a traditional design in the case of the optimal heat conductor and the adopted formulation for optimal thermal insulation.

Section 4.2 focuses on some applications concerning the sustainable design of building envelopes. The capabilities of the proposed formulation are firstly assessed in the achievement of optimal distributions of insulating material to mitigate the effect of thermal bridges. Subsequently, the design of modular elements for curtain walls is addressed, presenting layouts that minimize thermal transmittance in both the two-dimensional and the three-dimensional environment.

\subsection{Heat conduction vs. thermal insulation}

The Example 1.a) of Figure 1(L) is firstly investigated. A square lamina with side $5 m$ and unitary thickness is discretized by means of 10000 quadrangular elements, meaning that the same number of unknowns is considered to perform material distribution. A set of convective edges with coefficient $h_{c}=1 \mathrm{~W} /\left(\mathrm{m}^{2} K\right)$ is assigned along two opposite regions of the domain, i.e. the bold boundaries depicted in the figure. The ambient temperature $T_{a i}=1 \mathrm{~K}$ is enforced at the lower left corner of the lamina $\Gamma_{c i}$, while $T_{a o}=0 \mathrm{~K}$ is prescribed at the opposite upper right corner $\Gamma_{c o}$. No normal flux is allowed to cross the remaining part of the boundary $\Gamma_{f}=\Gamma \backslash \Gamma_{c}$, as straightforwardly achieved in the finite elements model with no additional enforcement. A similar problem was originally tackled 
in Iga et al. (2009) that considers the same boundary conditions on $\Gamma_{f}$, but prescribes an assigned temperature distribution on $\Gamma_{c i}$ and a fixed flux on $\Gamma_{c o}$. In both cases the aim is the activation of a heat flow that crosses the domain $\Omega$ between opposite corners. The geometry is firstly optimized according to Eqn. (5). The goal of the procedure is finding the distribution of the fraction $V_{f}=0.3$ of a conductive material with $k_{\rho=1}=100 \mathrm{~W} /(\mathrm{mK})$ in order to maximize the heat diffusivity within $\Omega$. The value $k_{\rho=0}=0.01 \mathrm{~W} /(\mathrm{mK})$ is assumed for the complementary phase, while a filter radius $r=1.5 d$ is implemented for the compliance sensitivities, being $d$ the reference dimension of the finite elements in the mesh. Figure 2(L) shows the achieved 0-1 optimal layout, denoting in black color the conductive fraction and in white color the complementary region. As expected from physical evidence and literature experiences the optimal design links the opposite boundaries $\Gamma_{c i}$ and $\Gamma_{c o}$ along the shortest track, i.e. the diagonal of the square domain.

The same geometry and boundary conditions are subsequently addressed in order to test the setting of Eqn. (6), providing the optimal thermal insulation between $\Gamma_{c i}$ and $\Gamma_{c o}$. The problem may be formulated as a two-phase optimization that looks for the optimal distribution of the fraction $V_{f}=0.3$ of an insulating material with $k_{\rho=0}=0.01 \mathrm{~W} /(\mathrm{mK})$, being $k_{\rho=1}=100 \mathrm{~W} /(\mathrm{mK})$ the conductivity coefficient of the complementary phase. Figure $2(\mathrm{R})$ shows the optimal $0-1$ layout. The black color denotes the insulating fraction, while the white region stands for the more conductive phase where $\rho=1$. Once again physical evidence validates the achieved design. To minimize the heat flux crossing the domain, the allowed percentage of insulating material $V_{f}$ is equally divided in two symmetric regions adjacent to $\Gamma_{c i}$ and $\Gamma_{c o}$, respectively. The optimal layout of insulating material intercepts the heat flux where its magnitude is expected to exhibit the largest values, i.e. in the corner zones. Each one of the boundaries between insulating and conductive material has a smooth shape that provides a uniform insulation with respect to the whole range of diffusion directions.

A variation of the above geometry is considered in Figure 1(R). The Example 1.b) investigates the modifications on the optimal layouts when larger convective edges are taken into account with respect to the previous case. Material properties, mesh refinement and allowed volume fraction are the same of the above simulations, while the amplitude of $\Gamma_{c}$ is enlarged to hold half of the domain perimeter. The formulation of Eqn. (5) is firstly adopted to maximize heat diffusion. The achieved solution is represented in Figure 3(L) which shows the arising of two main conductive channels that fully connect the opposite convective edges. Such a design maximizes the heat flux crossing the boundaries, while minimizing conductive resistance within the domain. Afterwards, the setting in Eqn. (6) is considered with the aim of solving the problem of optimal thermal insulation on the same geometry. The result is depicted in Figure 3(R). Two extremal black regions of insulating material fully intercept the heat flux spreading from $\Gamma_{c i}$ and to $\Gamma_{c o}$, thus confining most of the conductive phase in the central part of the domain. While the maximization of heat diffusivity requires conductive regions to connect the largest surface on the convective edges, its minimization mainly calls for a complete separation of the opposite exchanging boundaries by means of insulating material. In full agreement with the results of the Example 1.a), the optimization setting of Eqn. (6) distributes the volume fraction of insulating material $V_{f}$ in the regions where the heat flux exhibits its largest magnitude, i.e. in the vicinity of the corner zones. The thickness of the black regions is graded in order to achieve a uniform insulation all over the domain, thus explaining the non-straight boundaries of the insulating layers.

It must be remarked that both the conductive "stiffness" and the convective boundary 
conditions play an important role in the considered heat diffusion problems, as pointed out by the noticeable sensitivity of the achieved optimal designs presented in the Figures 2 and 3. This encourages the adoption of numerical methods in order to improve topological design in relevant engineering applications.

As detailed in Section 3.4 the proposed numerical implementation may be indifferently used to cope with two-dimensional of three-dimensional cases of design optimization. Having the aim of testing the brick-based optimization setting, the geometry and boundary conditions of the Example 1.a) are extended to the three-dimensional framework. To this purpose a cube with side $5 m$ is discretized by means of 62400 hexahedral elements and an equal number of density unknowns is considered. Two regions $\Gamma_{c i}$ and $\Gamma_{c o}$ are defined in the vicinity of the two opposite corners with respect to a main diagonal of the cube, thus inducing a three-dimensional heat flux. Within an iterative procedure based on a repeated set of finite elements analysis, a key issue that remarkably affects computational time is the choice of the steady-state solver. The equations arising from the linear systems in the two-dimensional case have been tackled via a direct sparse solver, while an iterative strategy based on the preconditioned conjugate gradient has been preferred in the three-dimensional case, because of the improved numerical performances with respect to brick-based models. One of the advantages of the use of ad hoc finite elements packages is the possibility of adopting different storage and coupled solutions strategies depending on the features of the considered model. Reference is made to API Manual (2005) for more details on the adopted numerical schemes.

The results of the three-dimensional optimization are shown in Figure 4. More in detail, the picture on the left refers to the problem for maximum heat conduction. The three-dimensional distribution of conductive material is denoted in black color and is the straightforward extension of the result presented in Figure 2(L). The optimal layout is a homogenous channel, free from undesired intermediate density regions, that links the opposite domain boundaries with assigned convective properties. Similarly, the right picture in Figure 4 corresponds to the three-dimensional design for thermal insulation represented in Figure 2(R). The black regions of insulating material are located in the immediate vicinity of the corners next to $\Gamma_{c i}$ and $\Gamma_{c o}$, while smooth spherical surfaces divide the material fractions. As remarked in the plane case, the boundaries between conductive and insulating phases are normal to the three dimensional main fluxes that cross the domain.

Numerical aspects of the comparison between the formulations of Eqns. (5) and (6) are finally addressed in Figure 5, that shows convergence diagrams for two- and threedimensional computations on the Example 1.a). Figure 5(L) refers to the minimization setting for optimal conduction, while Figure 5(R) addresses the maximization scheme for thermal insulation. The abscissa of the diagrams reports the number of iterations, while the ordinate plots the value of a non-dimensional objective functions, defined as $\mathcal{C} / \mathcal{C}_{\text {iter }=1}$, where $\mathcal{C}_{\text {iter }=1}$ is the thermal compliance computed at the first iteration. As detailed in Section 3.4, $\mathcal{C}_{i t e r=1}$ refers to $x_{i}=V_{f}, \forall i$, in the case of the design for thermal conduction, or to $x_{i}=\left(1-V_{f}\right), \forall i$, for the insulation problem. This provides, in both cases, a feasible starting point to the minimization algorithm. Each one of the graphs presented in Figure 5 shows smooth curves that are free from undesired numerical instabilities. The formulation for optimal insulation finds convergence a little bit earlier with respect to the case of optimal conduction, while a negligible increase in terms of number of iterations is observed for the brick-based runs. This encourages the use of three-dimensional simulations where this kind of modeling may consistently improve the physical description of the problem. 


\subsection{Applications to building thermal insulation}

One of the main challenges concerning the optimal design of a building envelope is the reduction of the energy losses due to undesired thermal bridges or to improper performances of external elements, such as curtain walls. To this purpose the thermal transmittance may be conveniently adopted as a measure to classify the elements belonging to the building envelope. This parameter, commonly denoted as $U$, corresponds to the heat flux that goes through a structural element with unitary surface in the presence of the difference $T_{a i}-T_{a e}=1 \mathrm{~K}$ between the ambient temperature on the internal side and the external one. The conduction-dominated heat transfer refers to steady-state conditions, meaning that $U$ is referred to the time unit. Concerning a pure mono-dimensional flux, a well-known formula for the computation of the thermal transmittance of a curtain wall made of $Z$ homogeneous layers with thickness $l_{i}$ and conductivity $k_{i}$ reads:

$$
U=\frac{1}{1 / h_{c i}+\sum_{i=1}^{Z} l_{i} / k_{i}+1 / h_{c e}} .
$$

The numbers $h_{c i}$ and $h_{c e}$ are flat parameters that take into account thermal exchanges on the free surfaces of the wall within the convective model of Eqn. (1). As introduced in Section 2, they refer to laminar flow conditions and are assumed to be independent on the internal structure of the building envelope, see e.g. ISO 6946:2007. For an assigned set of materials the above formula may be immediately exploited to design optimal curtain walls according to fixed constraints on the volume fractions of the insulating layers. The mono-dimensional flux allows in fact to work in terms of thickness of the layers, instead of a general description of the optimal topologies.

Unfortunately, Eqn. (9) may not be easily extended to many practical cases where more complex conditions involve two- or three-dimensional fluxes, thus calling for the adoption of ad hoc numerical methods. The formulation presented in Eqn. (6) is well-suited to address this kind of problems. The minimization of the thermal transmittance $U$ of a solid element may be tackled in fact as a minimization of the heat flux that is conducted through the domain, if the ambient temperature $T_{a i}=1 \mathrm{~K}$ is assigned to the convective internal side, i.e. $\Gamma_{c i}$, while $T_{a e}=0$ is enforced for the convective external edge, i.e. $\Gamma_{c e}$. Suitable coefficients $h_{c}$ may be assumed in agreement with engineering codes, see i.e. ISO 6946:2007. The following numerical studies refer to simulations of horizontal heat flows for which technical rules suggest the choice $h_{c i}=7.7 \mathrm{~W} /\left(\mathrm{m}^{2} \mathrm{~K}\right)$ and $h_{c e}=25 \mathrm{~W} /\left(\mathrm{m}^{2} \mathrm{~K}\right)$. Within the above framework Eqn. (6) is therefore applied to perform preliminary investigations on two common issues arising in the design of building envelopes for optimal thermal performances.

\subsubsection{Optimal design against thermal bridges}

Thermal bridging occurs when the uniformity of the building envelope is broken by any change in geometry or discontinuity of materials, which both may induce significantly higher heat losses with respect to their surroundings. Thermal bridges are characterized by the arising of multi-dimensional heat flows that cannot be adequately approximated by the one-dimensional model introduced in Eqn. (9). Technical rules suggest the adoption of numerical methods to take into account both the two-dimensional and the three-dimensional fluxes, see e.g. ISO 10211:2007. An accurate analysis of the heat problem is in fact the starting point for a careful designed aimed to the maximization of the insulation effectiveness.

Figure $6(\mathrm{~L})$ presents the case of a geometrical discontinuity of the building envelope due 
to a corner joint between two walls. The considered horizontal section may be modeled within a plane framework adopting the steady-state heat conduction equations introduced in Section 2. A set of convective-like boundary enforcements must be taken into account to impose indoor and outdoor conditions along $\Gamma_{c i}$ and $\Gamma_{c o}$, respectively. Two regions $\Gamma_{f}$ are also considered at a certain distance from the corner, to enforce a null heat flux through the sides perpendicular to the wall axes. With such a geometry one may easily notice that the convective length of the external edges is larger than the corresponding internal boundary. This induces an increased heat flux in the vicinity of the inner point of the corner, thus generating an undesired "cold" zone due to the arising of the thermal bridge. The formulation of Eqn. (6) is therefore applied to the design of the optimal topology for a volume fraction $V_{f}=0.3$ of insulating material with $k_{\rho=0}=0.025 \mathrm{~W} /(\mathrm{mK})$. The complementary phase has the conductivity coefficient of $k_{\rho=1}=0.25 \mathrm{~W} /(\mathrm{mK})$. It must be pointed out that the adoption of suitable convective-like boundary conditions is an essential requirement for the correct modeling of the thermal bridge. A simpler imposition of a fixed distribution of assigned surface temperature on $\Gamma_{c}$ would not be able to capture the "cold" zone at the inner point of the corner. The result of the performed optimization is shown in Figure 6(R), where the black region stands for the optimal topology of low-conductivity material. The maximization of the thermal compliance $\mathcal{C}$ provides an insulating layer with variable thickness that is located along the inner side of the corner. This intercepts the incoming flux while minimizing the overall length of the layer. As found in the previous examples, the boundary between conductive and insulating material lies perpendicular to the direction of the expected heat flux. In the vicinity of the corner, the incoming flux interests a larger surface and follows a longer track with respect to the extremal regions included in the model. According to Eqn. (9) the optimization procedure reduces the thickness of the black layer next to the inner point of the corner, thus providing an optimal uniform insulation all over the considered geometry. This also avoids the arising of any "cold" zone, i.e. undesired discontinuities in the distributions of the surface temperature on $\Gamma_{c i}$, in full agreement with well-known solutions of building engineering.

Another frequent thermal bridge is due to the discontinuity of materials that make the building envelope. Figure $7(\mathrm{~L})$ sketches the horizontal section of a homogeneous curtain wall $\left(k_{\rho=1}=0.25 \mathrm{~W} /(m K)\right)$ that is broken by a square concrete column $\left(k_{c o n}=1.25 \mathrm{~W} /(\mathrm{mK})\right)$. The pillar, i.e. the colored region in the figure, is a weak point that noticeably affects the thermal performances of the considered portion of the building envelope. The high conductivity of concrete with respect to the remaining part of the wall induces a preferred path for the heat that crosses the wall according to a twodimensional flow. This creates a "cold" zone on the inner side of the pillar along to a remarkable heat loss that cannot be handled by means of the purely mono-dimensional Eqn. (9). The formulation of Eqn. (6) is therefore implemented to find the optimal distribution of a volume fraction $V_{f}=0.2$ of insulating material $\left(k_{\rho=0}=0.025 \mathrm{~W} /(\mathrm{mK})\right)$, such that the overall thermal transmittance is minimized. The position of the concrete column is fixed and the optimization domain is reduced to the remaining part of the geometry. Figure 7(R) shows that the optimal topology consists of a black layer of insulating material that completely separates the inner pillar from the outer boundary of the building envelope. As found before, this result is in full agreement with common practice of building engineering. It also adds a few information that gives some insights into the problem. Looking at Figure $7(\mathrm{R})$ one may easily notice that the insulating layer is a little bit thicker, close to the column. This compensates the gap between the values $k_{\rho=1}$ and $k_{\text {con }}$, towards a uniform degree of insulation along the wall. The achieved solution is 
an interesting alternative with respect to a straight layout located in the vicinity of the external side of the envelope. The optimal topology distributes the heat flows crossing the column on a wider area, thus increasing the conductive resistance and reducing the heat losses in the critical regions.

A discontinuity of geometry and material is finally addressed in Figure $8(\mathrm{~L})$, where the effects of a concrete corner pillar are investigated. The thermal properties of the materials are the same of the previous example. Once again the adopted formulation has the aim of finding the distribution of the volume fraction $V_{f}=0.2$ of insulating material that minimizes thermal transmittance in the portion of the building envelope. Figure $8(\mathrm{R})$ presents the result of the optimization, showing an external low-conductive layer that surrounds the pillar to completely separate the two sides of the wall. The position of the column does not allow for the achievement of a topology similar to that found in Figure 6(R) and the optimal design is mainly derived from the solution presented in Figure $7(\mathrm{R})$. The insulating layer is adjacent to the external edge of the pillar and moves towards the outer sides of the envelope next to the extremal parts of the domain. This allows for an improved spreading of the high heat flows that arise in the corner region within the considered domain.

The above examples show that the proposed simulations are able to provide solutions for thermal bridges that are in good agreement with practical results that are well-known in building engineering. The adoption of numerical methods may be therefore exploited to optimize insulation layouts in more complex cases that are affected by discontinuities of geometry and material.

\subsubsection{Optimal design of a modular curtain wall}

Let consider the relevant horizontal section of a modular curtain wall made by precast blocks with thickness $30 \mathrm{~cm}$, as presented in Figure 9. Colored regions stand for the structural material, e.g. a concrete mixture with a thermal conductivity of $k_{\text {con }}=1.25 \mathrm{~W} /(\mathrm{mK})$. White zones correspond to a design domain that may be filled with an assigned volume fraction $V_{f}$ of well-insulating material $\left(k_{\rho=1}=0.025 \mathrm{~W} /(\mathrm{mK})\right)$, along with a complementary amount $1-V_{f}$ made of a poorer phase $\left(k_{\rho=0}=0.25 \mathrm{~W} /(m K)\right)$. The mono-dimensional scheme of Eqn. (9) cannot be straightforwardly adopted in the considered investigation, since the thermal bridge due to the discontinuity of material induces two-dimensional flows within the domain. As known from practice and also found in the previous numerical simulations, an effective solution to this kind of thermal bridging should be the adoption of an insulating layer to cover one of the external sides of the precast blocks. This would create an effective thermal separation between indoor and outdoor conditions. The considered example focuses on the manufacturing of the modular blocks as presented in Figure 9 and does not take into account any additional finish. In such a case the thermal bridge cannot be completely eliminated, but should be at least mitigated by the adoption of a suitable design of the insulating phase within concrete reinforcements. To this purpose the two-phase topology optimization problem of Eqn. (6) is solved, having the aim of minimizing heat diffusivity for an assigned set of volume fractions $V_{f}$ of well-insulating material. Figure 9 presents two possible fixed layouts of the modular structure of the wall. The picture on the left is denoted as Example 3.a) and addresses a standard distribution of transversal concrete reinforcements within the section. The picture on the right is denoted as Example 3.b) and refers to a finer network of structural material. Symmetry conditions call for no normal flux on the short boundaries, while the temperature difference between the internal and the external ambient is enforced by a set of suitable convective-like edges, as described above. 
The Example 3.a) is firstly addressed and four optimal designs for different amounts of allowed $V_{f}$ are presented in Figure 10. The pictures report in black the distribution of well-insulating material, while white zones refer to the more conductive phase. In all the achieved designs the well-insulating material is suitably distributed in order to avoid any continuity in the direction of the main flux between the two remaining fractions. The thickness of the black zones remarkably grows in the vicinity of the T-junctions made of conductive structural material, thus reducing the heat flux flowing into the wall around these regions. Figure 10 clearly shows that the shape of the boundaries between black and white zones is remarkably affected by the choice of $V_{f}$. The optimal designs for $V_{f}=0.2$ and $V_{f}=0.8$ mainly consist of two straight black stripes which wrap the concrete members located perpendicularly to the main flux. For $V_{f}=0.4$ and $V_{f}=0.6$ the optimal solutions adopt three layers of insulating materials. To investigate the effects of the adopted $V_{f}$ on the thermal performances of the achieved layouts, the convergence curves of the performed optimizations are compared in Figure 11. The plot presents nondimensional transmittances that are defined as the ratio $U / U_{V_{f}=0}$, where $U_{V_{f}=0}$ refers to a design only made of the fixed structural reinforcements and the poor-insulating material. The curves show a smooth trend towards different converge plateaux which point out that the thermal transmittance of the optimal layout for $V_{f}=0.2$ is approximately 1.5 times the value of the solution for $V_{f}=0.8$.

The presence of a multi-material domain with an articulated geometry considerably affects the heat fluxes that are generated by the conduction-dominated heat transfer. The Example 2.b) is hence addressed to show the effect of a variation in the location of transversal structural reinforcements through the wall section. The achieved optimal layouts are depicted in Figure 12 for the cases $V_{f}=0.2, V_{f}=0.4, V_{f}=0.6$ and $V_{f}=0.8$ and may be straightforwardly compared with the relevant designs in Figure 10. The compact shape of the fixed layout of the wall defines a more stable topology among structural reinforcements. In each one of the designs reported in Figure 12 three stripes of black well-insulating material separate two zones made of the white phase. Comparing Figure 10 and Figure 12 one may also notice the differences in terms of the curvature that defines the shapes of the boundaries between the well-insulating fraction and the poor-insulating phase.

The values of thermal transmittance of the achieved optimal designs $\left(U_{O p t}\right)$ are also reported in Table 1, along with the transmittance of a relevant set of reference designs $\left(U_{S t d}\right)$ that share with the considered optimal topologies the parameter $V_{f}$. In this "standard" class of topologies the well-insulating material is assumed to be massed in a single layer with constant thickness in the center of the domain, while the poor-insulating fraction involves the remaining part of the available geometry. For an assigned $V_{f}$, the adoption of the optimal topology may provide a gain in terms of transmittance up to $13 \%$ with respect to the assumed "standard" layouts, see columns $\Delta$ in the tables. The same results are also presented in Figure 13, where the depicted curves interpolate the transmittances $U_{O p t}$ and $U_{S t d}$ versus the volume fraction $V_{f}$, for both Examples 3.a) and 3.b). The graphs allow to emphasize the benefit of the adoption of topology optimization schemes within a performance-based framework. Let prescribe a target performance for the thermal transmittance of the precast wall of Figure $9(\mathrm{R})$, e.g. $0.6 \mathrm{~W} /\left(\mathrm{m}^{2} K\right)$. The assumed "standard" design would require $V_{f}=0.6$ to meet the fixed requirement, while the optimal topology needs only $V_{f}=0.4$. This means that the adoption of the proposed optimization setting would allow for a saving of about $30 \%$ in terms of the volume fraction of the well-insulating (and probably more expensive) material.

Another two-dimensional numerical study refers to the geometry in Figure 14, that is 
used to investigate the optimal topology of the modular curtain wall of Figure 9(L) in the case of a corner junction and a fixed volume fraction $V_{f}=0.6$. Looking at Figure 14(R) one may easily notice that the main layout in the extremal regions of the modeled domain is not so different with respect to the modular three-layered topology reported in Figure 10 for the same $V_{f}$. According to the result in Figure 6(R), most of the wellinsulating material is located in the vicinity of the inner edge of the considered domain, thus intercepting the heat flux along the shortest convective boundary. Next to the corner, the optimal design has to tackle both effects of the discontinuity of geometry and the heat losses due to concrete reinforcements between the inner and the outer sides of the wall. The solution consists of a local variation of the modular topology described above, i.e. the introduction of a well-insulating region that wraps concrete reinforcements with decreasing thickness towards the external boundary of the wall connection. The poorinsulating material is massed far from the inner point of the corner, as similarly found in Figure 6(R).

An effective approach to the investigation of the modular layout of an optimal curtain wall should take into account a three-dimensional description of the heat fluxes. Precast blocks are generally separated by mortar layers that introduce an additional discontinuity of materials in the vertical direction. To this purpose the geometry of the Example 3.b) is extruded in the third dimension and two layers with $k_{c o n}=1.25 \mathrm{~W} /(\mathrm{mK})$ are additionally modeled on the top and the lower sides of the considered domain. The boundaries $\Gamma_{c i}$ and $\Gamma_{c o}$ refer to the faces located on the internal and the external surfaces of the wall, respectively, while no normal flux is allowed on the remaining four edges due to symmetry reasons. Figures 15 and 16 present relevant horizontal and vertical sections of the optimal modular layouts achieved in the cases $V_{f}=0.6$ and $V_{f}=0.8$. The pictures depict only the fraction of well-insulating material along with structural reinforcements/mortar. Figure 15 is generated with a cutting plane located at an equal distance from the upper and lower mortar layers, while the section plane of Figure 16 crosses the finite elements model in the middle of the cavity between two adjacent vertical reinforcements. The achieved design of Figures 15(R) and 16(R) exhibit many similarities with the corresponding two-dimensional layout reported in Figure 12 for $V_{f}=0.8$. New solutions may also arise in the three-dimensional field, as in the case of Figures 15(L) and $16(\mathrm{~L})$, where a four-layered topology is introduced instead of the simpler layout achieved in Figure 12 for $V_{f}=0.6$. The resort to the brick-based modeling allows in fact for a complete description of the geometry to be optimized, taking into full account the effective three-dimensional physical framework.

\section{Conclusions}

A numerical scheme for the topology optimization of conduction-dominated problems has been implemented to address issues as the optimal design of thermal insulation in building engineering. The method maximizes a suitable form of the thermal compliance, coping with heat diffusivity within a design domain that has assigned convective-like boundary conditions. A rational approximation scheme is used to interpolate the thermal conductivity on the density unknown, handling a two-phase topology optimization. The numerical scheme has been implemented within a general-purpose finite elements code and coupled to a gradient-based minimization algorithm via an application programming interface. Such an implementation takes full advantage of the modeling features of the finite elements code and exploits the numerical capabilities of the embedded large sparse 
solvers, also in the three-dimensional framework.

A few preliminary examples have been presented to assess the main features of the method and to compare maximization and minimization procedures from the point of view of achieved results and convergence features. The methodology has been subsequently tested within its natural field of application, i.e. the minimization of thermal transmittance for the optimal design of the building envelope. The optimal insulation against the arising of thermal bridges has been firstly investigated in the case of discontinuities of geometry or material within the body of the envelope. Afterwards, the preliminary design of modular blocks for curtain walls has been tackled, providing optimal topologies both in the two-dimensional and in the three-dimensional framework. A comparison with reference layouts has also been presented in order to discuss the benefit due to the adoption of the implemented numerical method.

The proposed formulation is able to generate optimal layouts that are in good agreement with theoretical and practical results that are well-known from building engineering. It must be remarked, however, that the achieved topologies are preliminary designs that do not take into account production requirements or other kind of engineering constraints. The use of the proposed methodology may be therefore intended as a support to the designer in the cases where the arising of complex heat fluxes may not be tackled via classical methods for mono-dimensional propagation.

\section{References}

Allaire G., 2002, Shape optimization by the homogenization method, Berlin, Heidelberg, New York, Springer-Verlag.

Bendsøe M.P. and Kikuchi N., 1988, Generating optimal topologies in structural design using a homogeneization method, Comput. Methods Appl. Mech. Eng., 71(2), 197-224.

Bendsøe M.P. and Sigmund O., 1999, Material interpolation schemes in topology optimization Arch. Appl. Mech., 69, 635-654.

Bendsøe M.P. and Sigmund O., 2003, Topology optimization - Theory, methods and applications, Berlin, Heidelberg, New York, Springer-Verlag.

Bruggi M., 2009, Generating strut-and-tie patterns for reinforced concrete structures using topology optimization, Computers \& Structures, 87(23-24), 1483-1495.

Bruns T.E., 2007, Topology optimization of convection-dominated, steady-state heat transfer problems, International Journal of Heat and Mass Transfer, 50, 2859-2873.

Bruyneel M., Duysinx P., 2005, Note on topology optimization of continuum structures including self-weight design, Struct. Multidisc. Optim., 29 4, 245-256.

Buttazzo G., 1988, An optimization problem for thin insulating layers around a conducting medium, Boundary control and boundary variations. Lecture notes in computer science, Springer, 100, 91-95.

Cherkaev A., 2000, Variational Methods for Structural Optimization, Berlin, Heidelberg, New York, Springer-Verlag.

Cho S. and Choi J.Y. , 2005, Efficient topology optimization of thermo-elasticity problems using coupled field adjoint sensitivity analysis method, Finite Elements Anal. Design, 41, 1481-1495.

Diaz A.R. and Benard A., 2003, Topology optimization of heat-resistant structures, Proceedings of ASME 2003 Design Engineering Technical Conferences, Chicago, Illinois.

Donoso A. and Sigmund O., 2004, Topology optimization of multiple physics problems modelled by Poissons equation, Lat. Am. J. Sol. Struct., 1(2), 169-189. 
Eschenauer H.A. and Olhoff N., 2001, Topology Optimization of Continuum Structures - A Review, Applied Mechanics Reviews, 54, 331-390.

Evgrafov A., Maute K., Yang R.G., Dunn M.L., 2008, Topology optimization for nanoscale heat transfer, Int. J. Num. Meth. Eng., 77(2), 285-300.

Gao T., Zhang W.H., Zhu J.H., Xu Y.J., Bassir D.H., 2008, Topology optimization of heat conduction problem involving design-dependent heat load effect, Finite Elements in Analysis and Design, 44, 805-813.

Gersborg-Hansen A., Bendsøe M.P., Sigmund O., 2006, Topology optimization of heat conduction problems using the finite volume method, Struct. Multidisc. Optim., 31, 251-259.

Gosz M.R., 2006, Finite element method: applications in solids, structures, and heat transfer, CRC Press, Taylor and Francis, Boca Raton.

Habbal A., Petersson J., Thellner M., 2004, Multidisciplinary topology optimization solved as a Nash game, Int. J. Num. Meth. Eng., 61(7), 949-963.

Iga A., Nishiwaki S., Izui K., Yoshimura M., 2009, Topology optimization for thermal conductors considering design-dependent effects, including heat conduction and convection, Int. J. of Heat and Mass Transfer, 52, 2721-2732.

Li Q., Steven G.P., Querin O.M., Xie Y.M., 1999, Shape and topology design for heat conduction by evolutionary structural optimization, Int. J. Heat Mass Transfer, 42, 3361-3371.

Li Q., Steven G.P., Xie Y.M., Querin O.M., 2004, Evolutionary topology optimization for temperature reduction of heat conducting fields, Int. J. Heat Mass Transfer, 47, 5071-5083.

Munoz E., Allaire G., Bendsøe M.P., 2007, On two formulations of an optimal insulation problem, Struct. Multidisc. Optim., 33(4-5), 363-373.

Sigmund O., 2001, A 99 line topology optimization code written in Matlab, Struct Multidisc Optim, 21, 120-127.

Sigmund O. and Petersson J., 1998, Numerical instabilities in topology optimization: A survey on procedures dealing with checkerboards, mesh-dependencies and local minima, Struct. Multidisc. Optim., 16(1):68-75.

Stolpe M. and Svanberg K., 2001, An alternative interpolation scheme for minimum compliance optimization, Struct. Multidisc. Optim., 22, 116-124.

Svanberg K., 1987, Method of moving asymptotes - A new method for structural optimization, Int. J. Num. Meth. Eng., 24(3), 359-373.

Zhou M. and Rozvany G.I.N., The COC algorithm, part II: Topological, geometry and generalized shape optimization, 1993, Comput. Meth. Appl. Mech. Eng., 106, 1-26.

Zhuang C.G., Xiong Z.H., Ding H., 2007, A level set method for topology optimization of heat conduction problem under multiple load cases, Comput. Methods Appl. Mech. Eng, 196, 1074-1084.

Zhuang C.G., Zhenhua X., Ding. H., 2010, Topology optimization of multi-material for the heat conduction problem based on the level set method, Engineering Optimization, 42(9), 811-831.

ISO 6946:2007. Building components and building elements Thermal resistance and thermal transmittance - Calculation method.

ISO 10211:2007. Thermal bridges in building construction - Heat flows and surface temperatures - Detailed calculations.

API Manual, 2005, Documentation for the Straus7 Application Programming Interface, Edition 6.a, API Release 2.3, Strand7 Pty Ltd. 

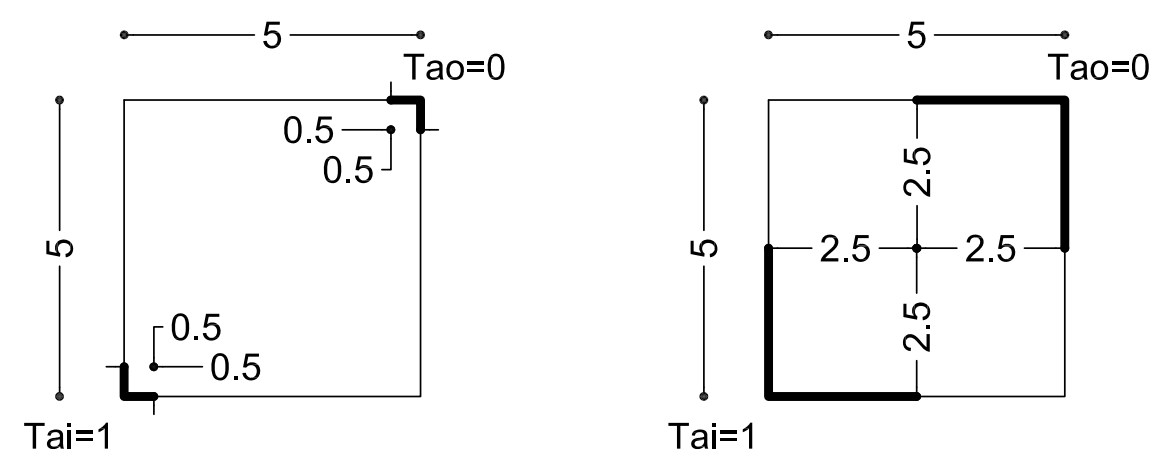

Figure 1. Example 1. Geometry and boundary conditions for Example 1.a)(L) and Example 1.b)(R) (dimensions in $m$, temperatures in $K$ ).
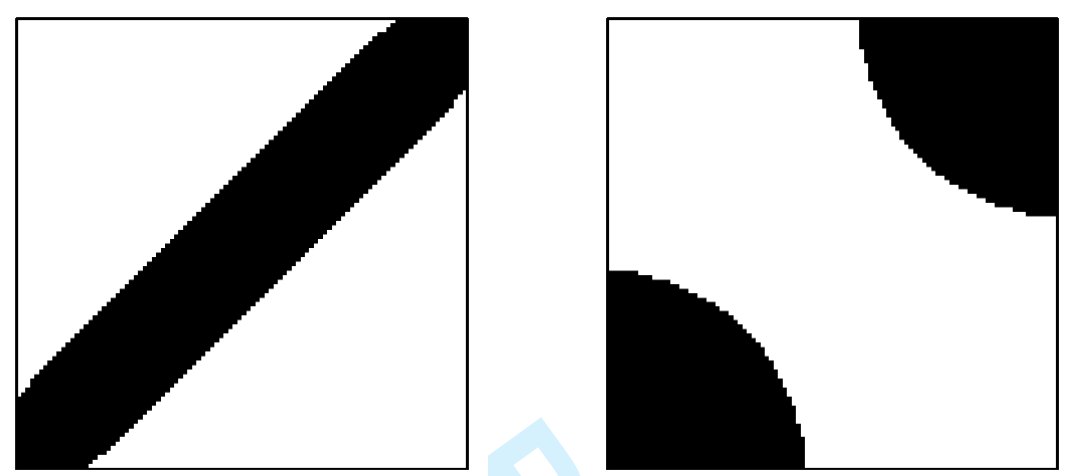

Figure 2. Example 1.a). Optimal two-dimensional designs for the heat conductor (conductive material in black)(L) and the thermal insulator (insulating material in black)(R): $V_{f}=0.3$.
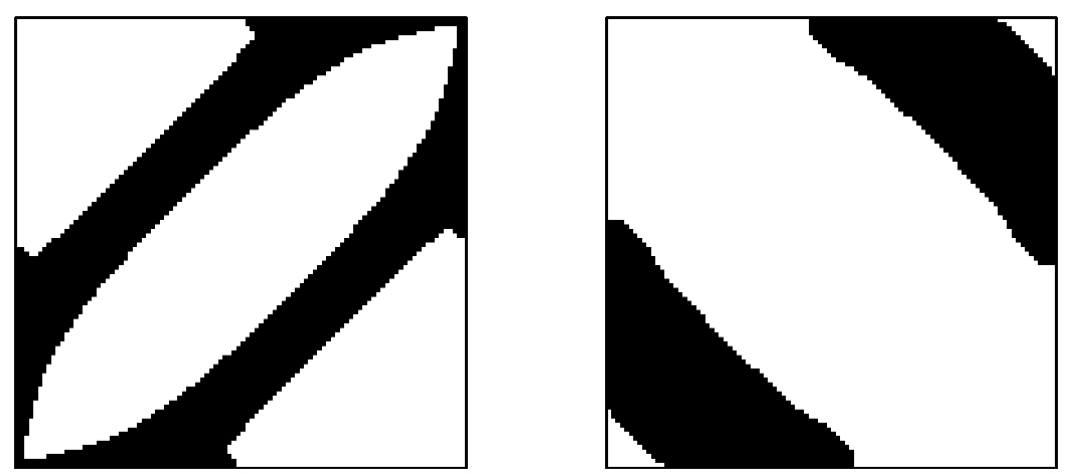

Figure 3. Example 1.b). Optimal two-dimensional designs for the heat conductor (conductive material in black)(L) and the thermal insulator (insulating material in black) $(\mathrm{R}): V_{f}=0.3$. 
1

3

4

5

7

8

9

10

11

12

13

14

15

16

17

18

19

20

21

22

23

24

25

26

27

28

29

30

31

32

33

34

35

36

37

38

39

40

41

42

43

44

45

46

47

48

49

50

51

52

53

54

55

56

57

58

59

60
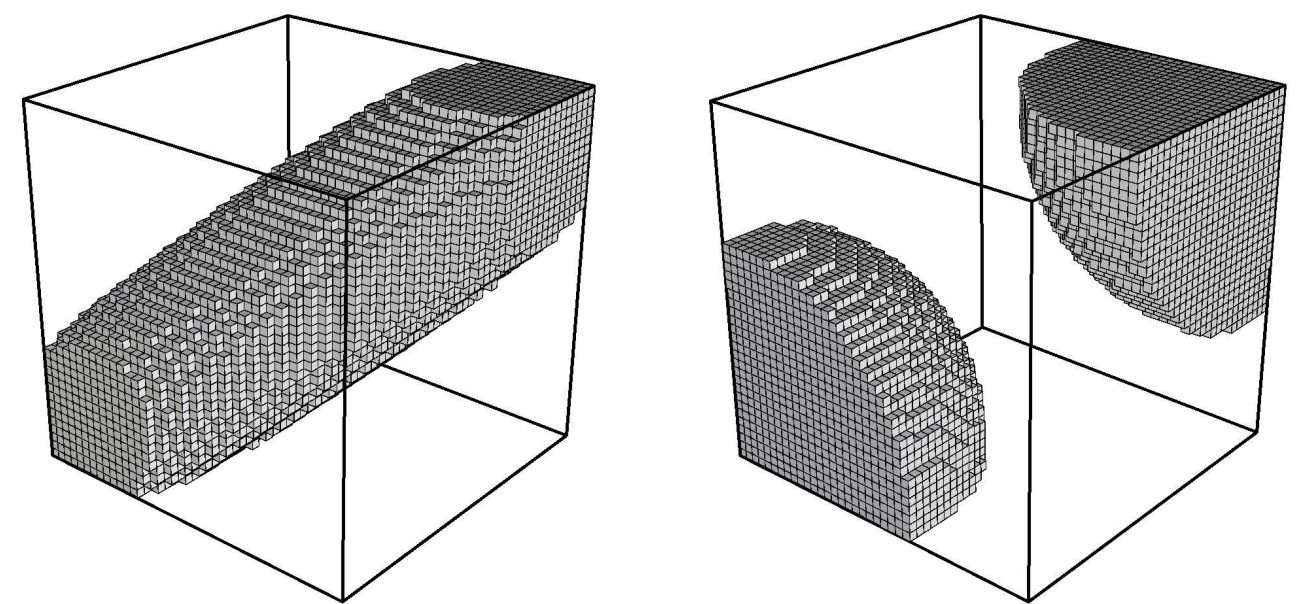

Figure 4. Example 1.a). Optimal three-dimensional designs for the heat conductor (conductive material in grey)(L) and the thermal insulator (insulating material in grey)(R): $V_{f}=0.3$.
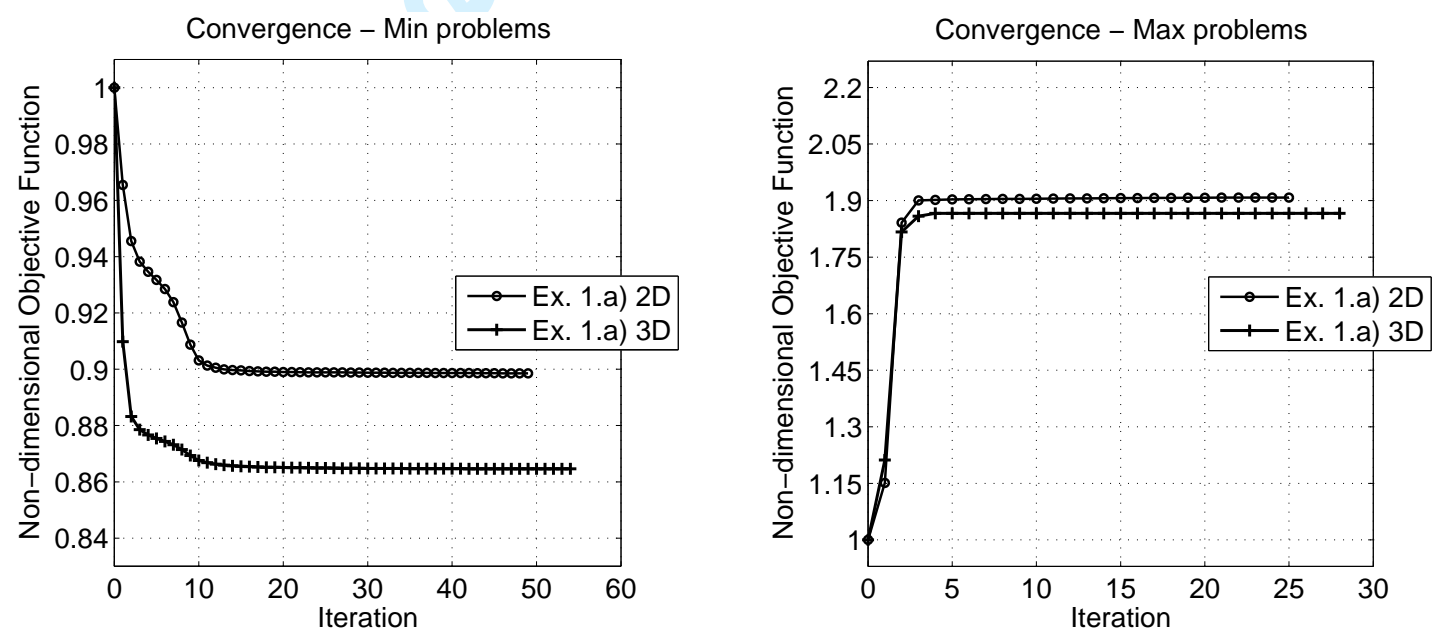

Figure 5. Example 1.a). Convergence diagrams for the heat conductor problem (L) and the optimization for thermal insulation (R). 
2

Figure 6. Example 2.a). Geometry (L) and optimal design (R) for maximum thermal insulation (dimensions in $\mathrm{cm}$, temperatures in $K$, insulating material in black): $V_{f}=0.3$.

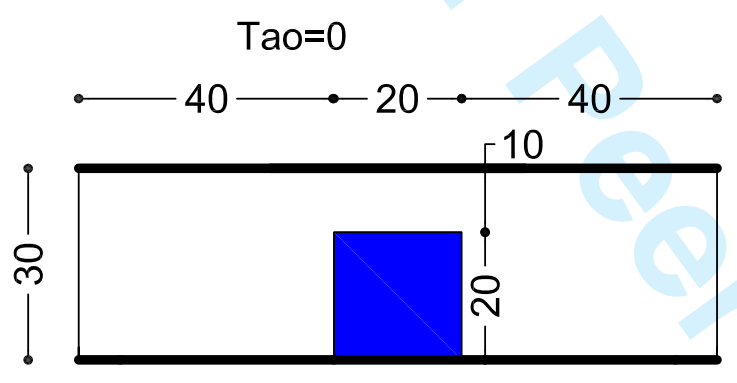

\section{Tai=1}

Figure 7. Example 2.b). Geometry (L) and optimal design (R) for maximum thermal insulation (dimensions in $\mathrm{cm}$, temperatures in $K$, insulating material in black): $V_{f}=0.2$.
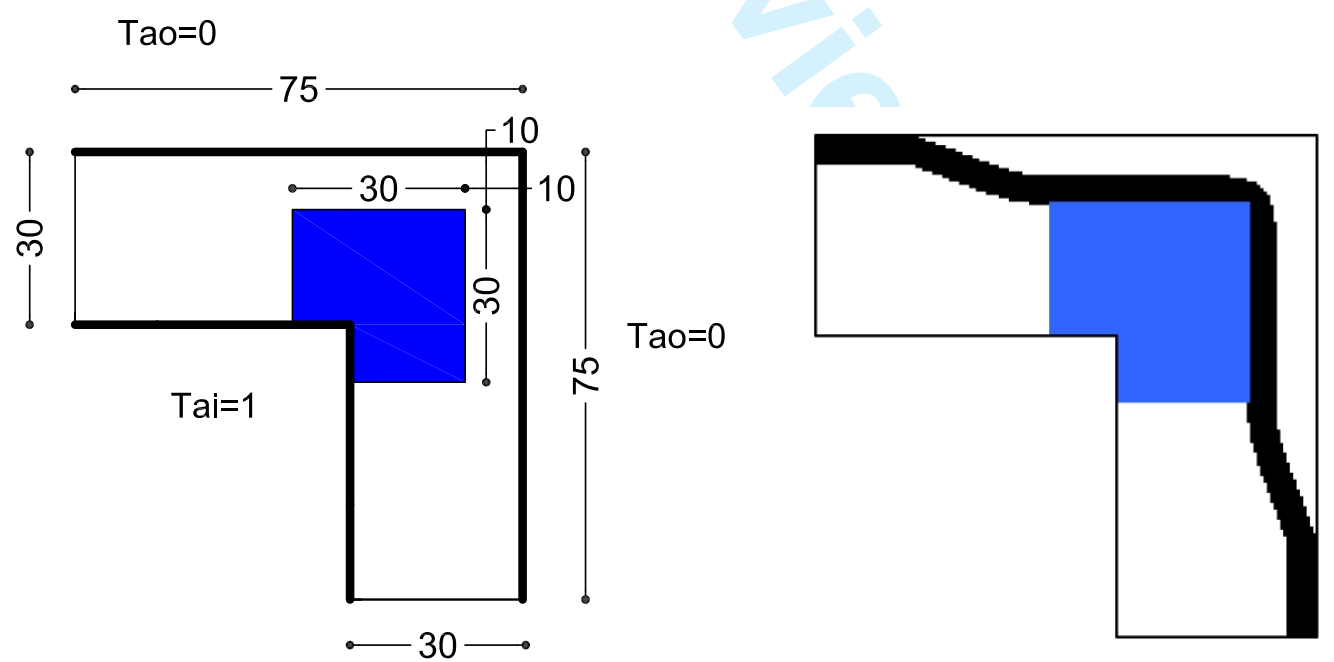

Figure 8. Example 2.c). Geometry (L) and optimal design (R) for maximum thermal insulation (dimensions in $\mathrm{cm}$, temperatures in $K$, insulating material in black): $V_{f}=0.2$. 

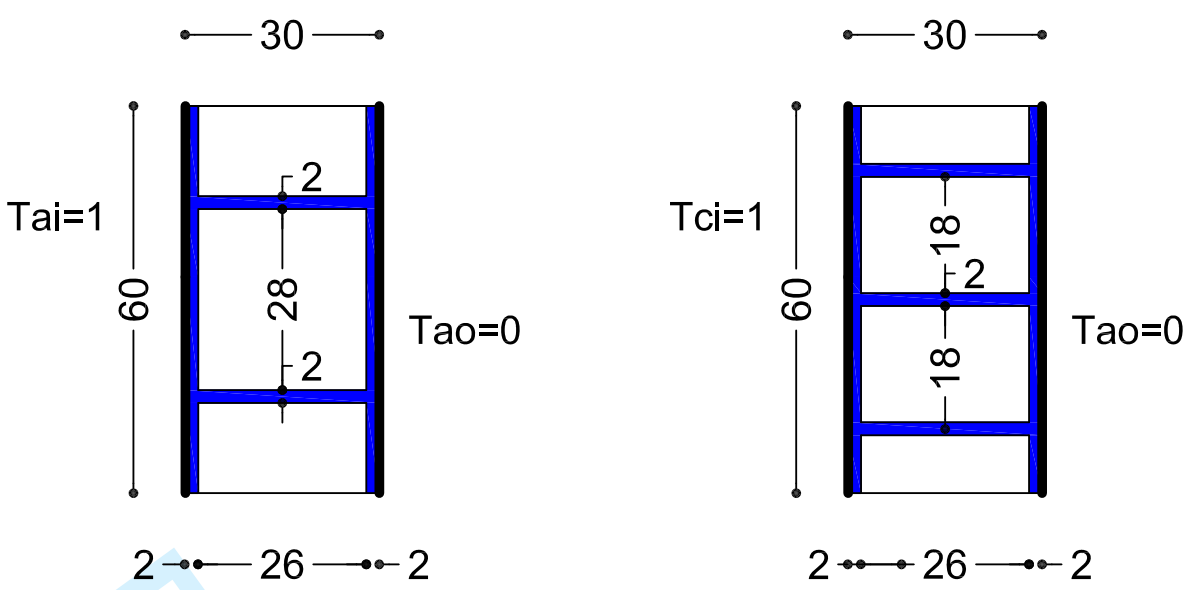

Figure 9. Example 3. Geometry and boundary conditions for Example 3.a)(L) and Example 3.b)(R) (dimensions in $\mathrm{cm}$, temperatures in $K$ ).
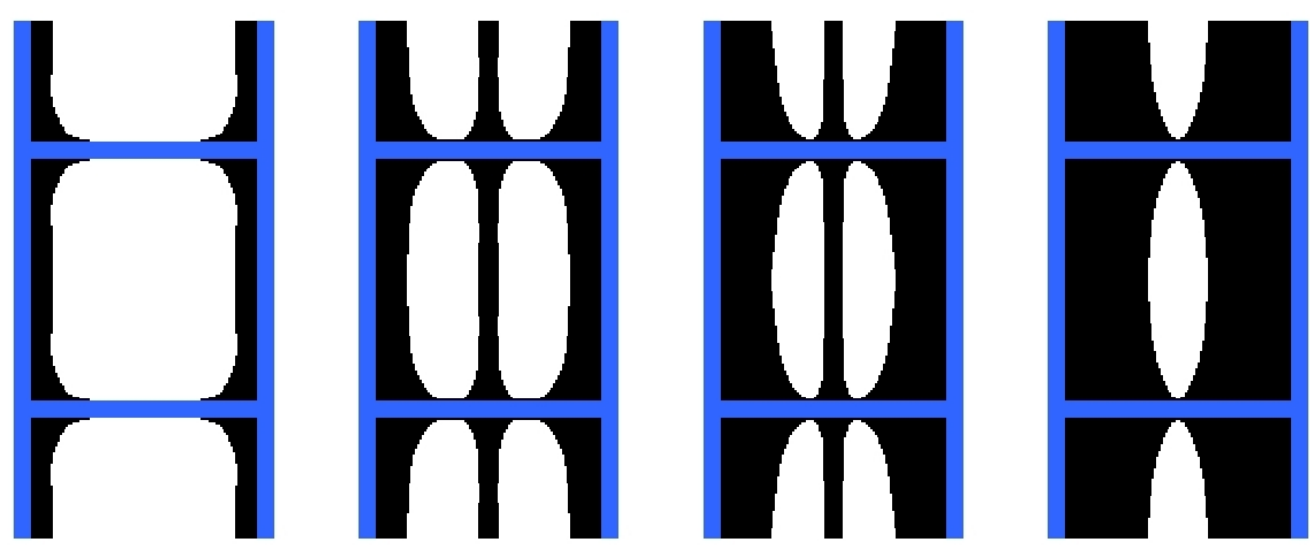

Figure 10. Example 3.a). Optimal designs for thermal insulation (well-insulating material in black): $V_{f}=0.2, V_{f}=0.4, V_{f}=0.6, V_{f}=0.8$.

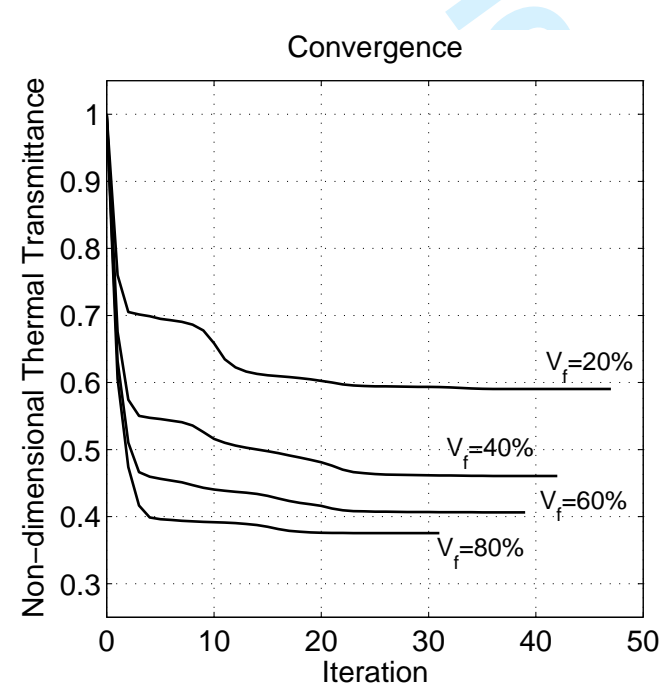

Figure 11. Example 3.a). Convergence diagrams for the optimal layouts in Figure 10. 

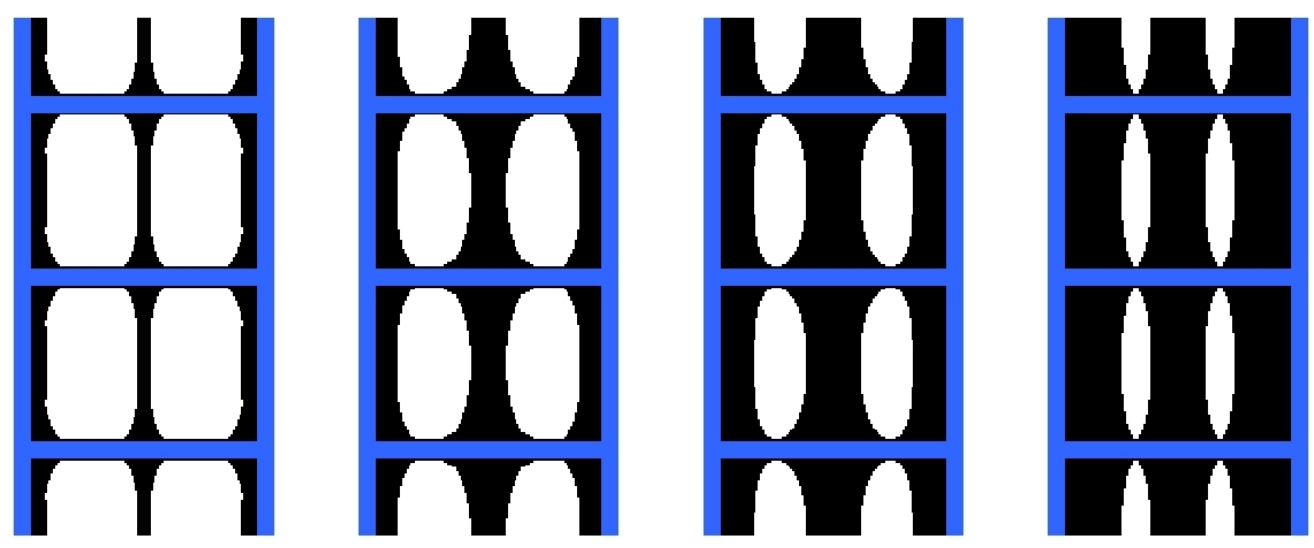

Figure 12. Example 3.b). Optimal designs for thermal insulation (well-insulating material in black): $V_{f}=0.2, V_{f}=0.4, V_{f}=0.6, V_{f}=0.8$.

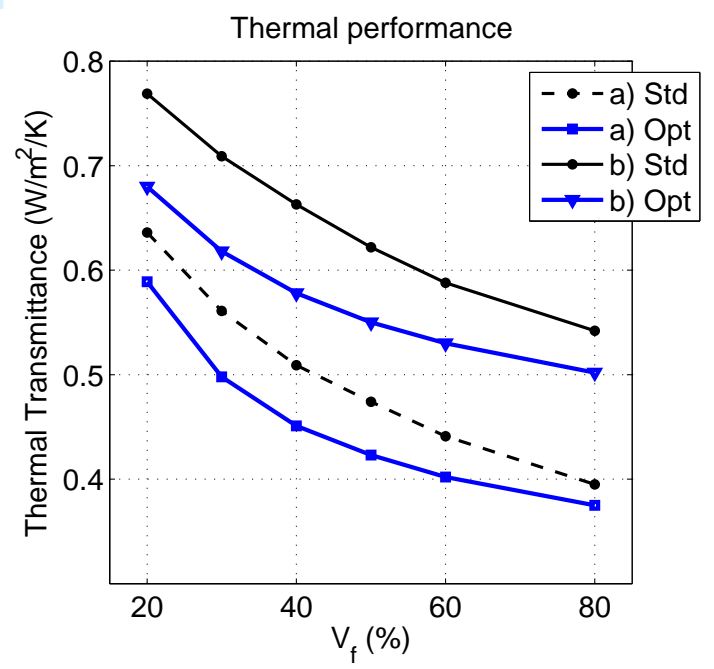

Figure 13. Example 3. Thermal performance of the optimal designs (Opt) as compared with reference solutions (Std) with the same volume fraction of well-insulating material $V_{f}$ : cases a) and $b$ ).
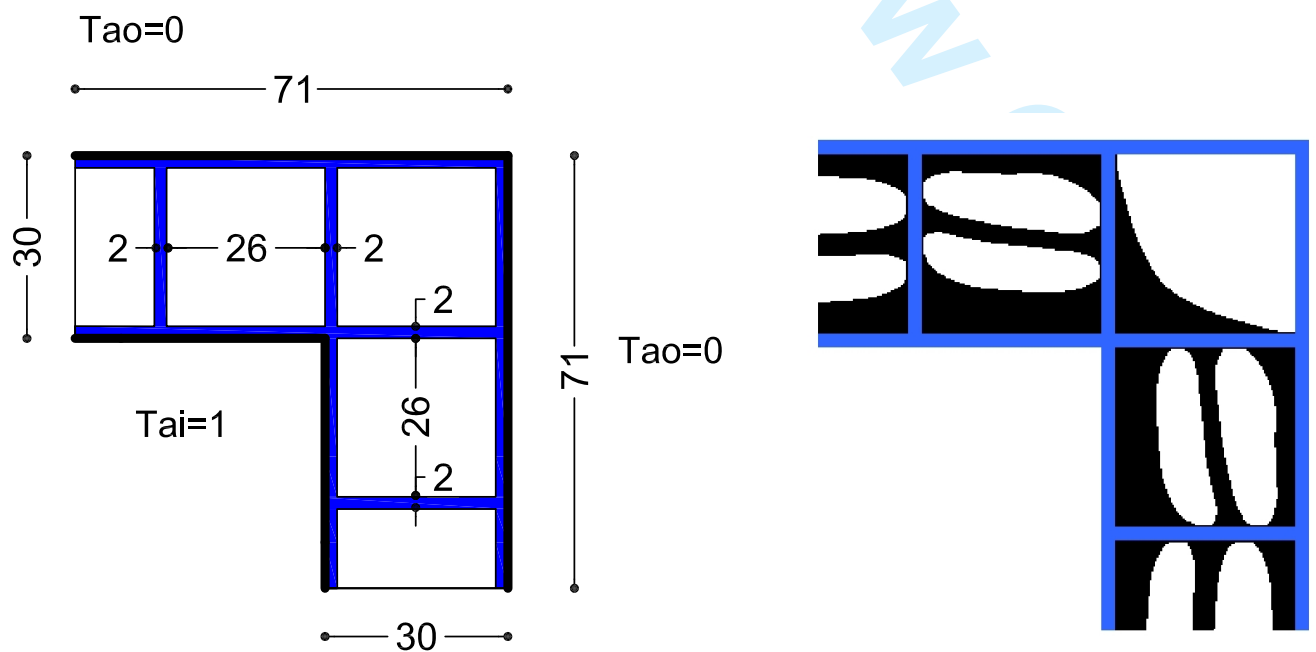

Figure 14. Example 3.c). Geometry (L) and optimal design (R) for maximum thermal insulation (dimensions in $\mathrm{cm}$, temperatures in $K$, well-insulating material in black): $V_{f}=0.6$. 
Figure 15. Example 3.b). Horizonal sections of the optimal three-dimensional designs for thermal insulation (well-insulating material in grey): $V_{f}=0.6(\mathrm{~L}), V_{f}=0.8(\mathrm{R})$.
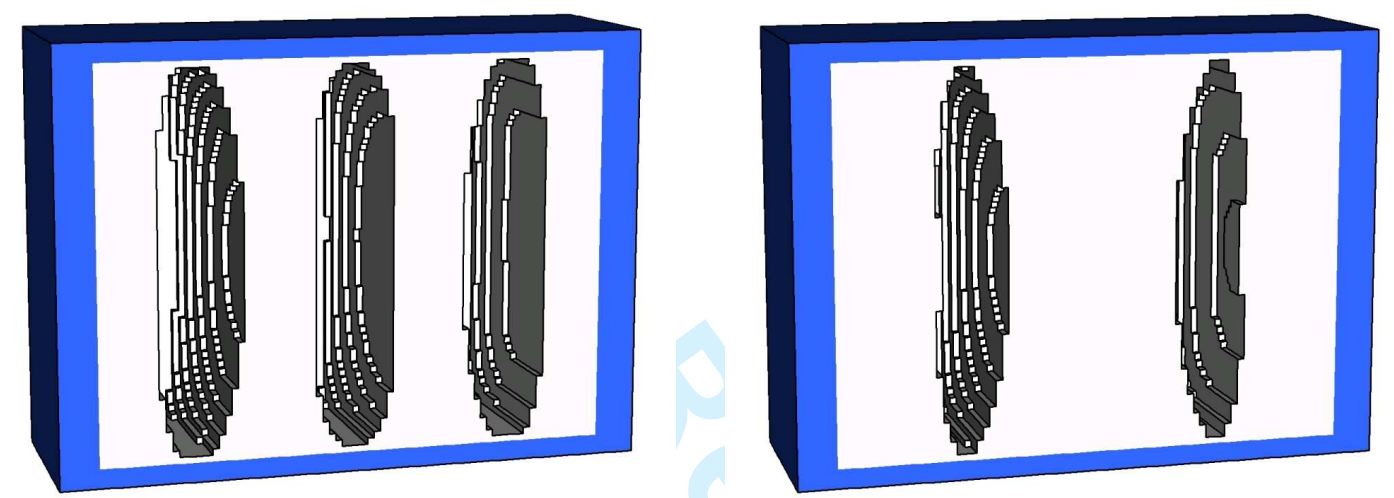

Figure 16. Example 3.b). Vertical sections of the optimal three-dimensional designs for thermal insulation (well-insulating material in grey): $V_{f}=0.6(\mathrm{~L}), V_{f}=0.8(\mathrm{R})$.

a)

\begin{tabular}{|c|c|c|c|}
\hline $\begin{array}{c}V_{f} \\
(\%)\end{array}$ & $\begin{array}{c}U_{S t d} \\
\left(W / m^{2} / K\right)\end{array}$ & $\begin{array}{c}U_{O p t} \\
\left(W / m^{2} / K\right)\end{array}$ & $\begin{array}{c}(\Delta) \\
(\%)\end{array}$ \\
\hline \hline 20 & 0,636 & 0,589 & 7,4 \\
40 & 0,509 & 0,451 & 11,5 \\
60 & 0,441 & 0,402 & 8,9 \\
80 & 0,395 & 0,375 & 5,1 \\
\hline
\end{tabular}

b)

\begin{tabular}{|c|c|c|c|}
\hline $\begin{array}{c}V_{f} \\
(\%)\end{array}$ & $\begin{array}{c}U_{S t d} \\
\left(W / m^{2} / K\right)\end{array}$ & $\begin{array}{c}U_{O p t} \\
\left(W / m^{2} / K\right)\end{array}$ & $\begin{array}{c}(\Delta) \\
(\%)\end{array}$ \\
\hline \hline 20 & 0,680 & 0,769 & 11,5 \\
40 & 0,578 & 0,663 & 12,8 \\
60 & 0,530 & 0,588 & 9,8 \\
80 & 0,502 & 0,542 & 7,4 \\
\hline
\end{tabular}

Table 1. Example 3. Thermal transmittance of the optimal designs $\left(U_{O p t}\right)$ as compared with reference solutions $\left(U_{S t d}\right)$ with the same volume fraction of well-insulating material $V_{f}$ : case a) $(\mathrm{L})$ and case b) $(\mathrm{R})$. 


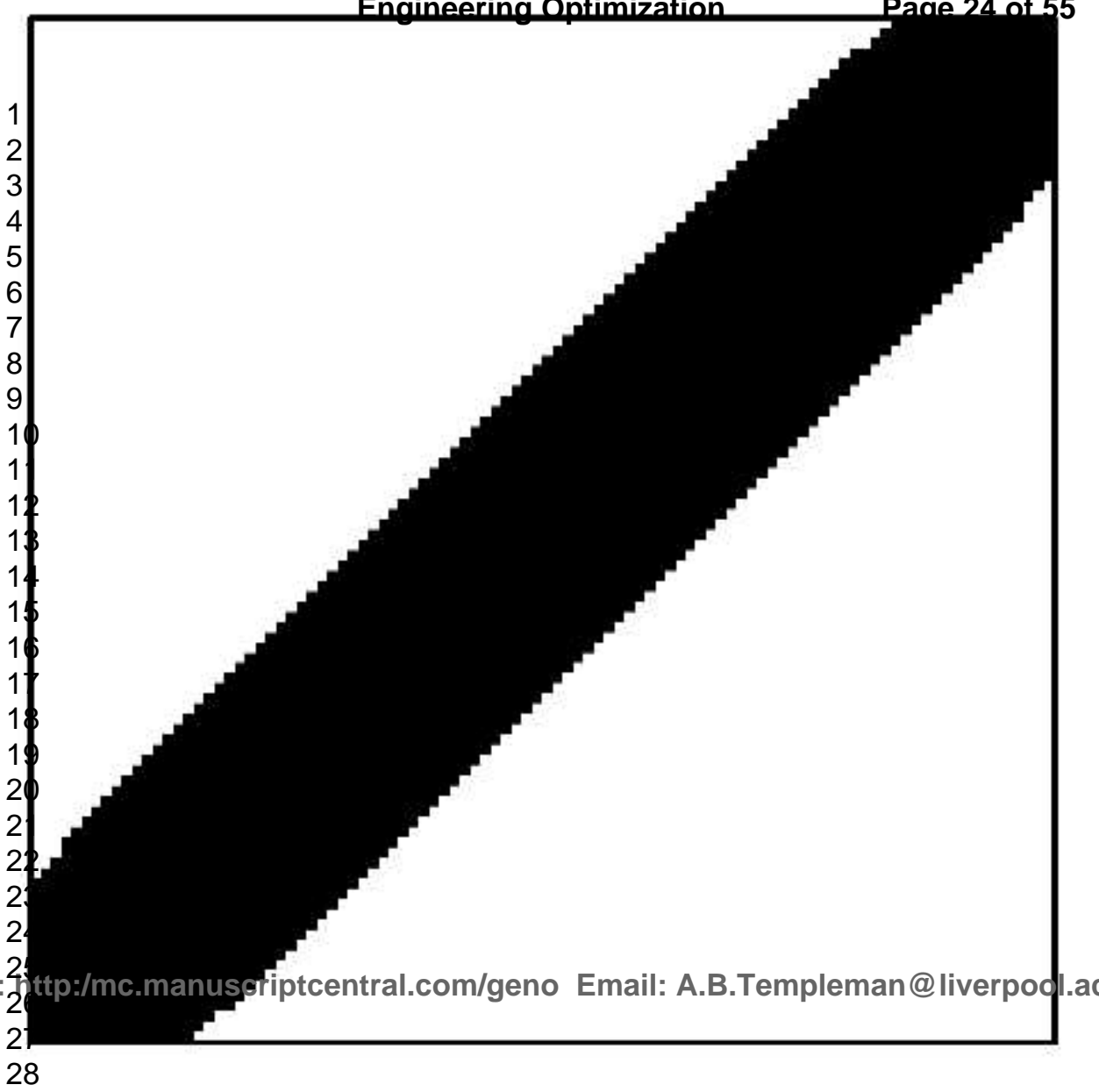


5

6

7

8

9

1

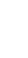

$$
1
$$

1

1
1
2
2
2
2
2

- 2

2

28 
Engineering Ontimization Pace 26 of 55

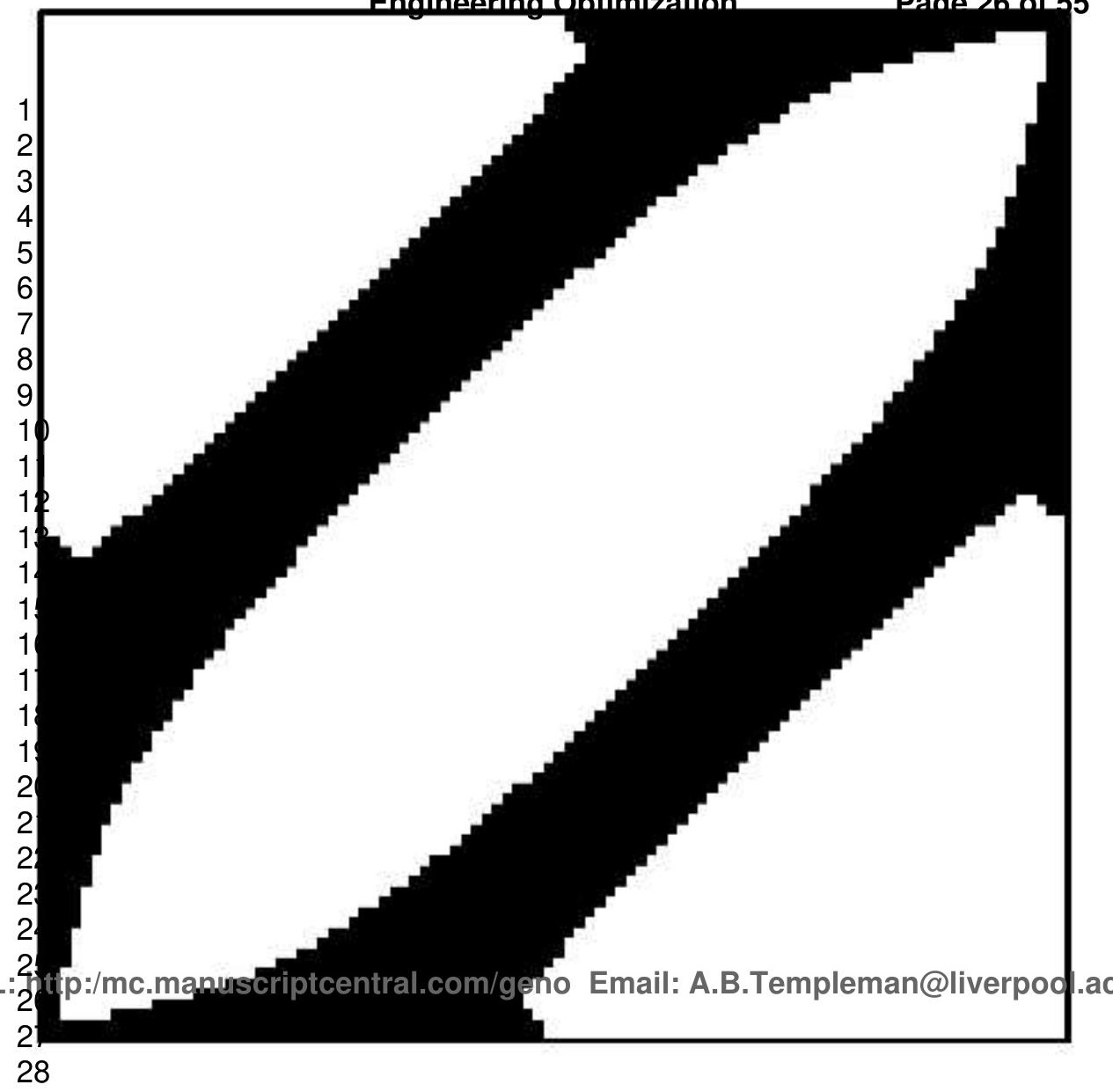




\section{Page 27 of 55}

Engineering Optimization

1
2
3
4
5
6
7
8
9
10
11
12
13
14
15
16
17
18
19
20
21
22
23
24
25
26
27
28
29
30
31
32
33
34
35
36
37
38
39
40
41
42
43
44

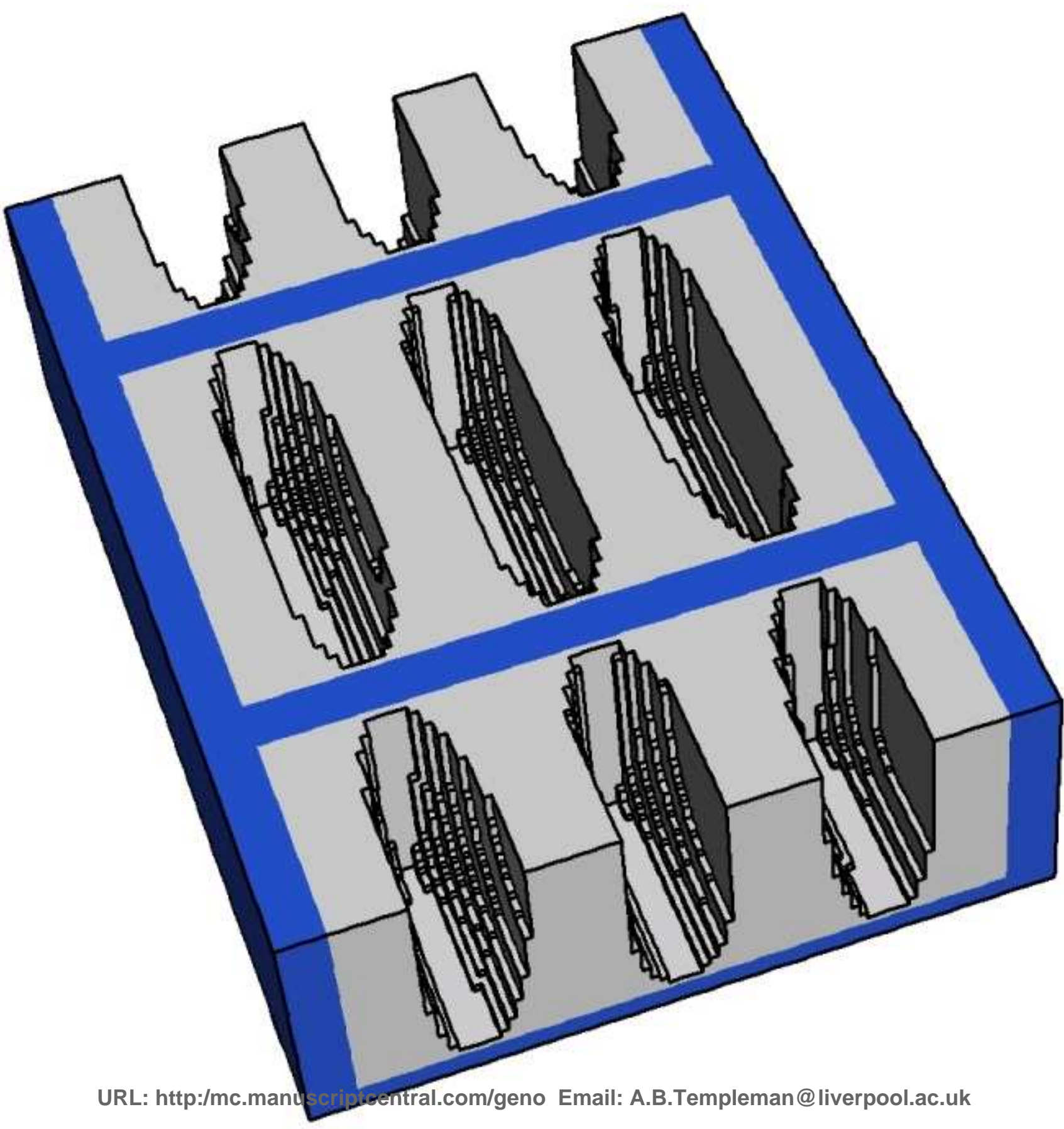




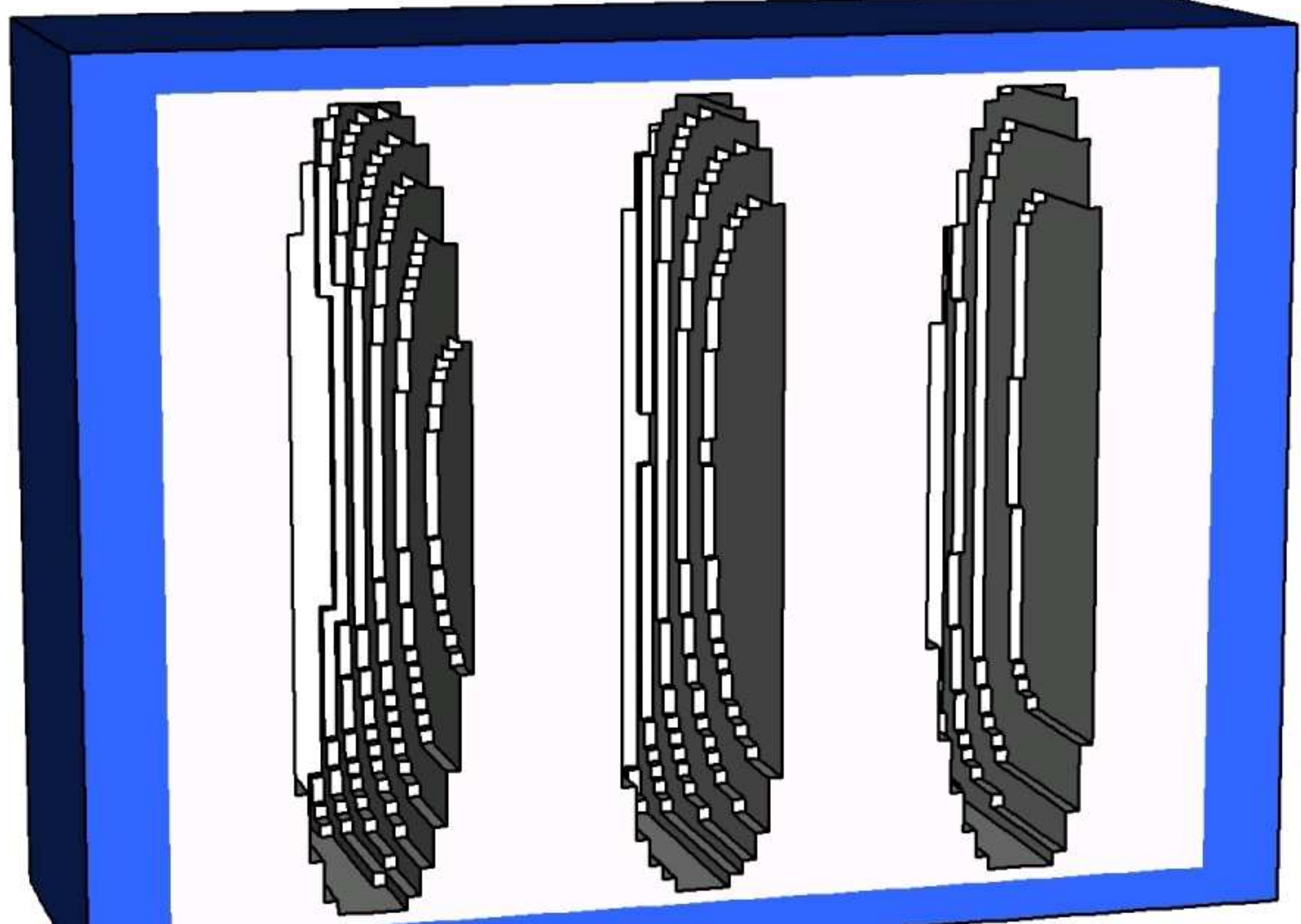

M-ritanuscriptcentral.com/geno Email: A.B.Templeman@liverpool.ac.uk 


\section{Page 29 of 55 \\ Engineering Optimization}

1
2
3
4
5
6
7
8
9
10
11
12
13
14
15
16
17
18
19
20
21
22
23
24
25
26
27
28
29
30
31
32
33
34
35
36
37
38
39
40
41
42
43
44

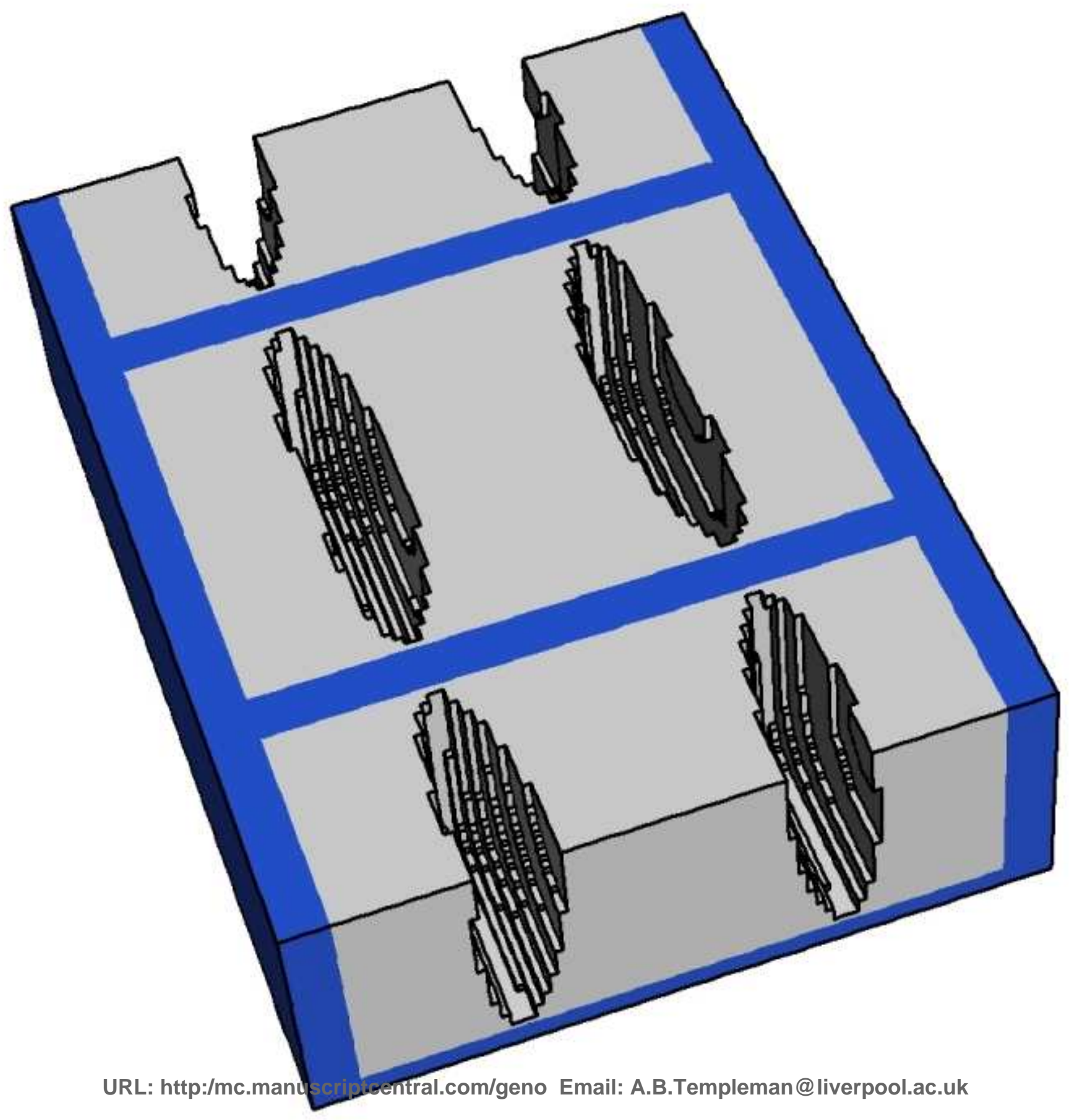

1 3 4 5 6
7 8 9 10 11 12 13 14 15 16 17 18 19

20

21

22
23 24 25 26 27 


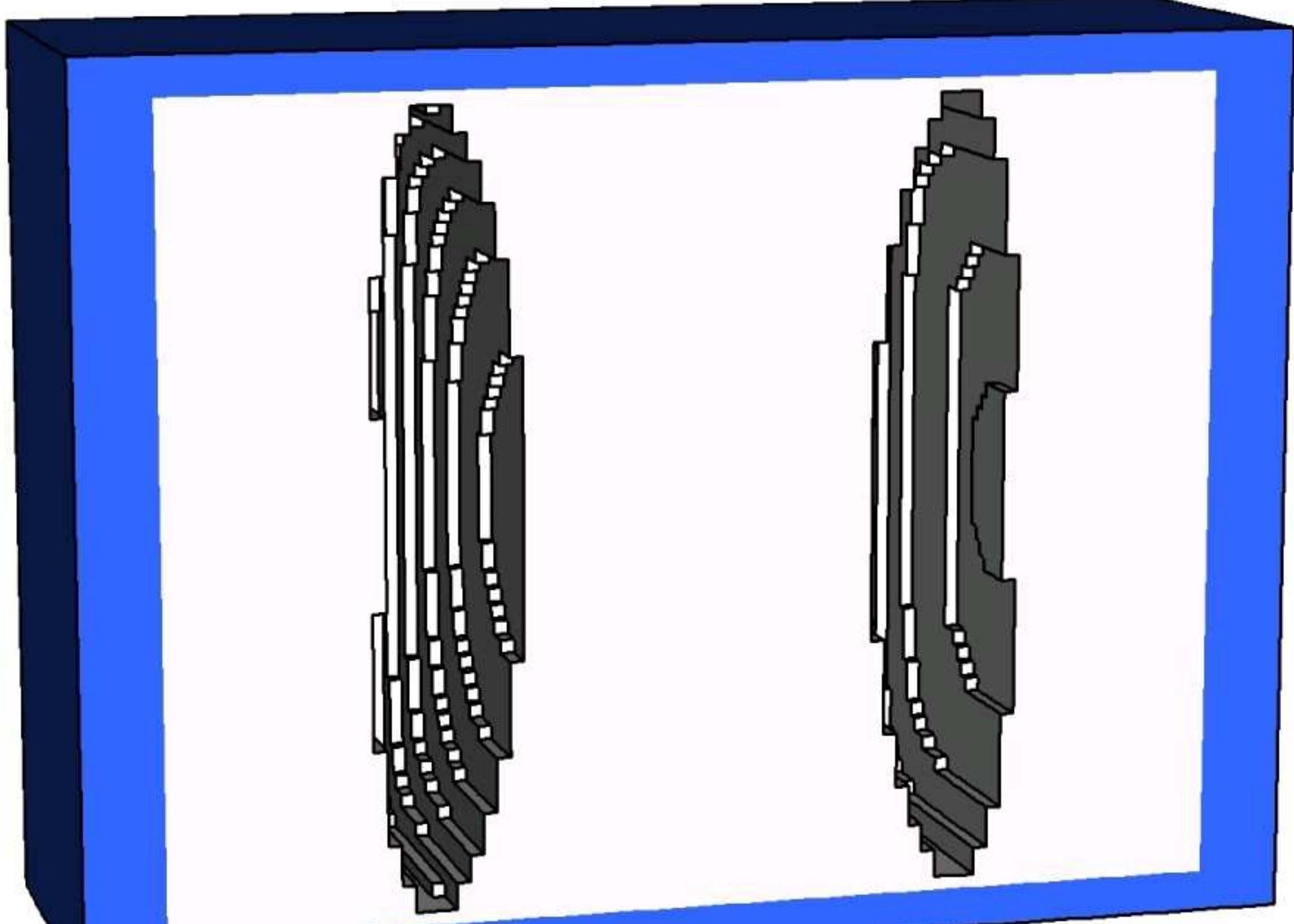




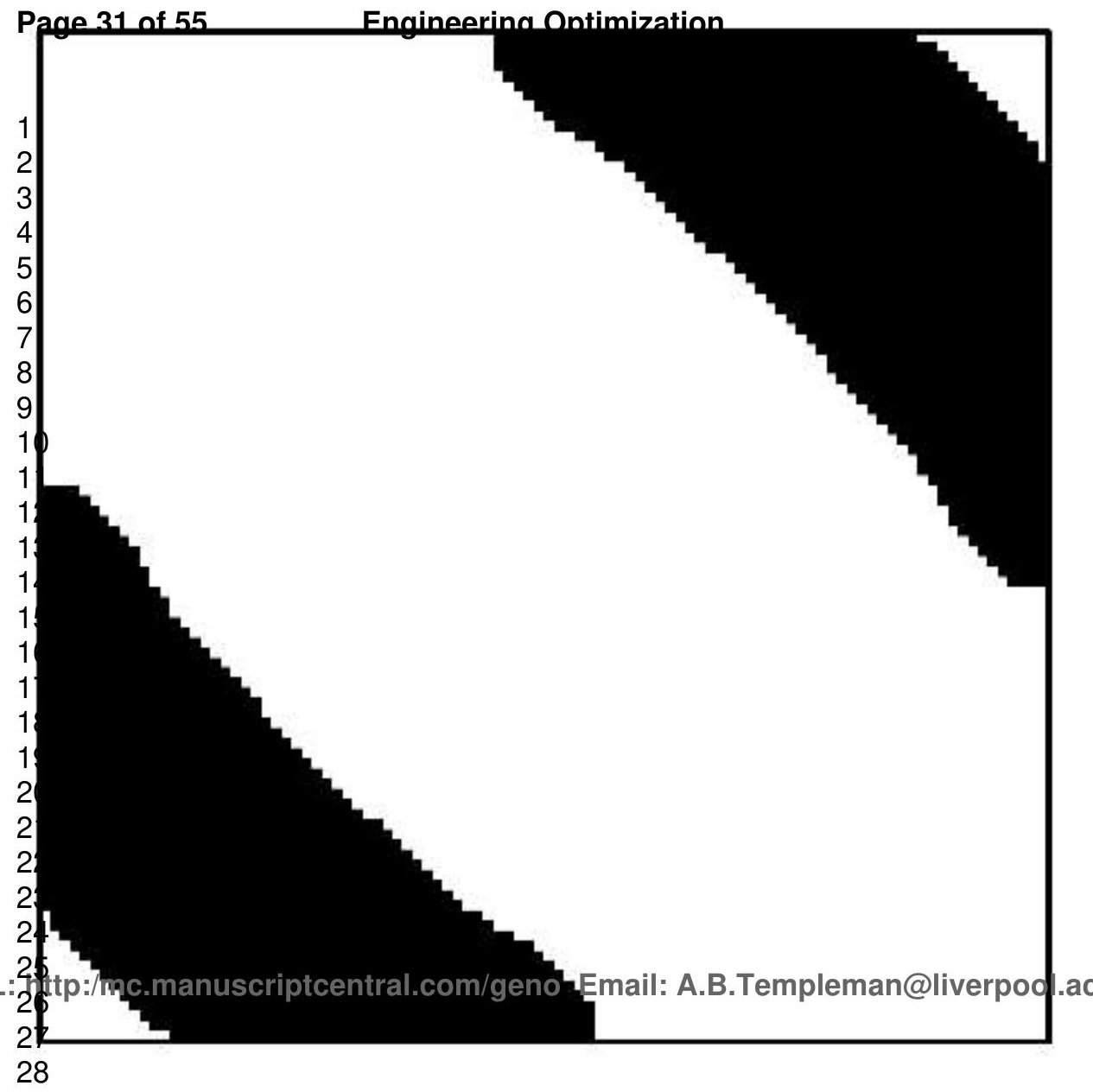




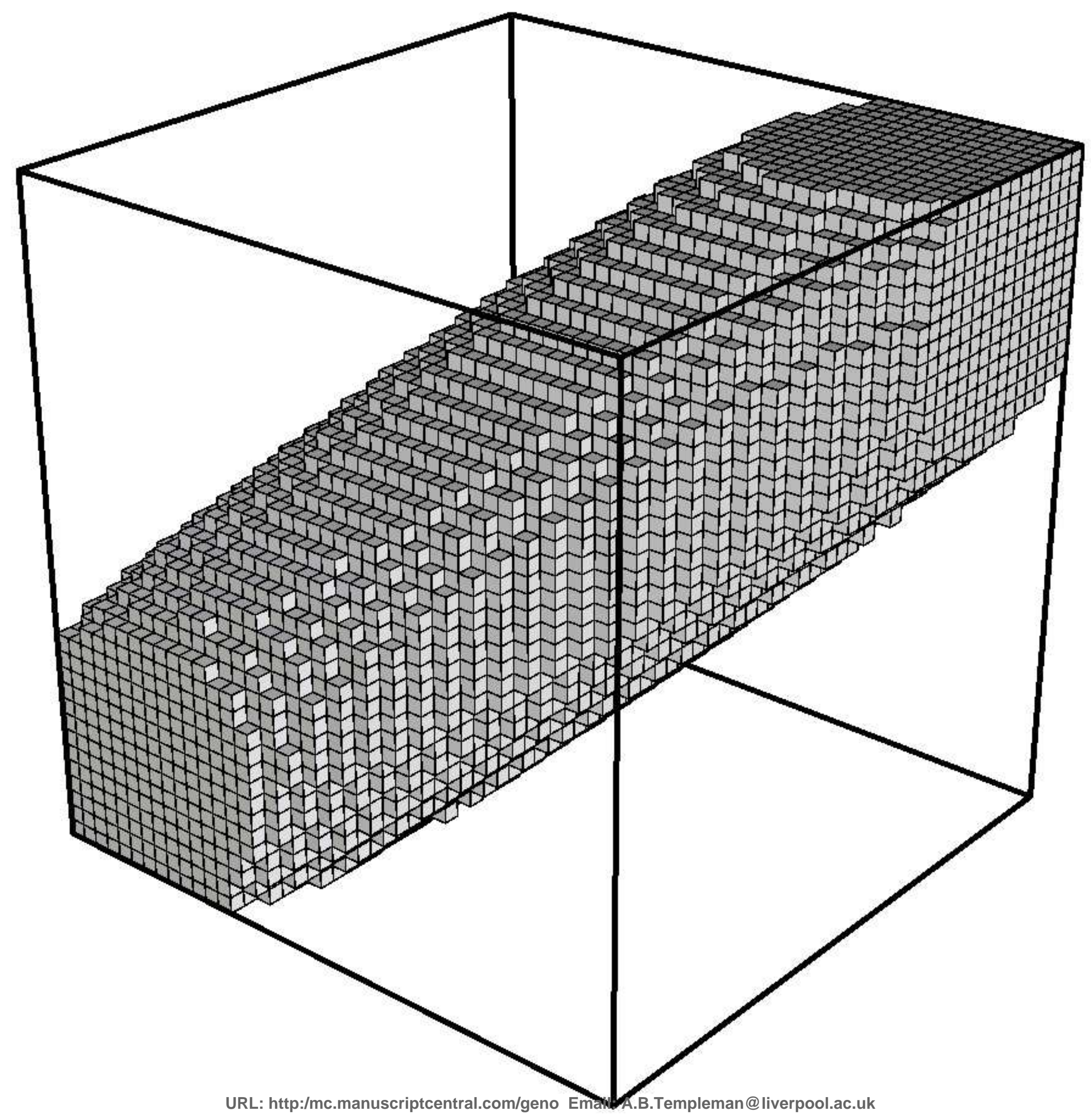




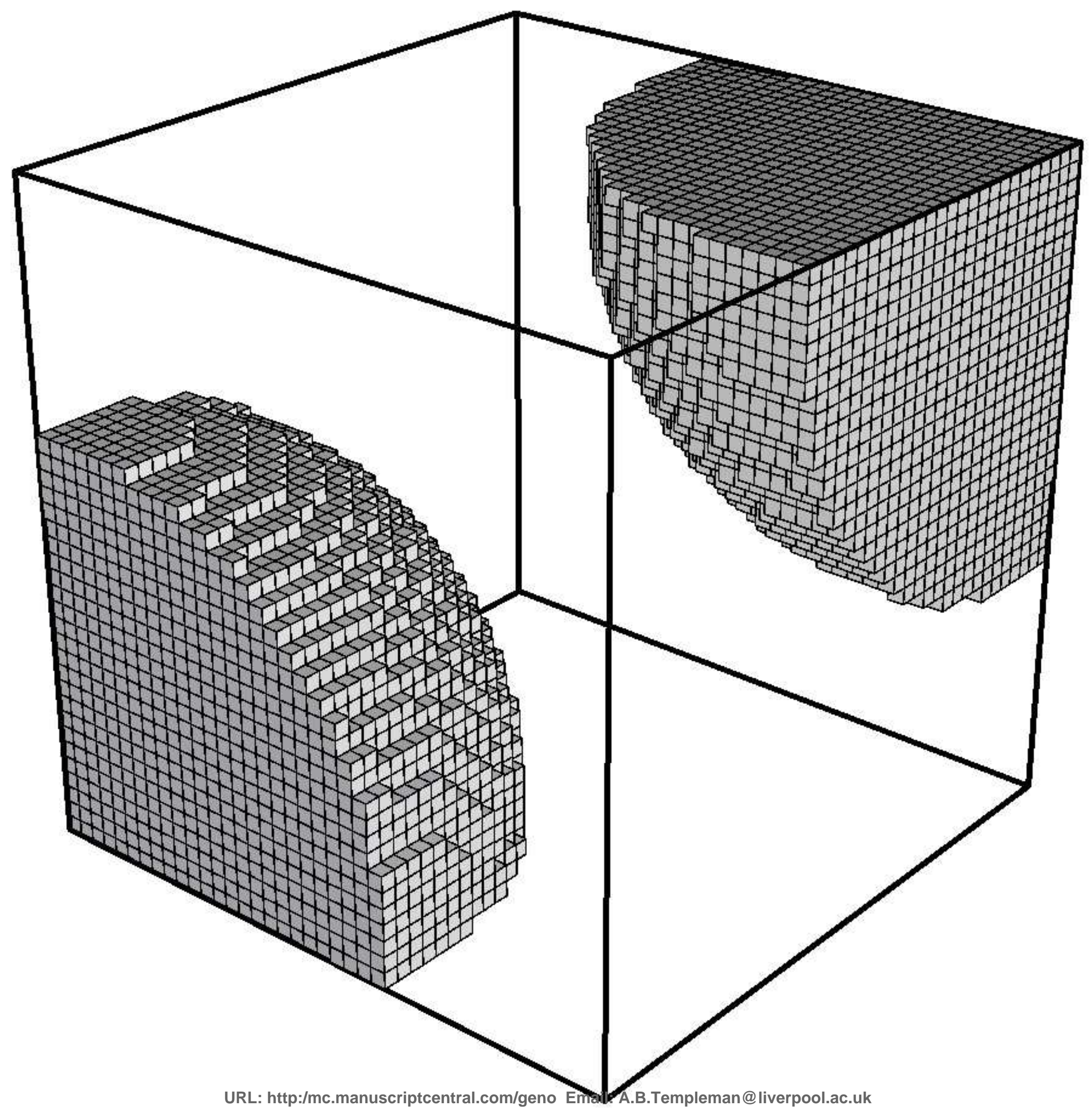


Engineering Optimizalliage_34 of 55

1
2
3
4
5
6
7
8
9

10

11

12

13

14

15

16

17

18

19

20

21

22

23

24

25

ung ri entral.com/geno Email: A.B.Te

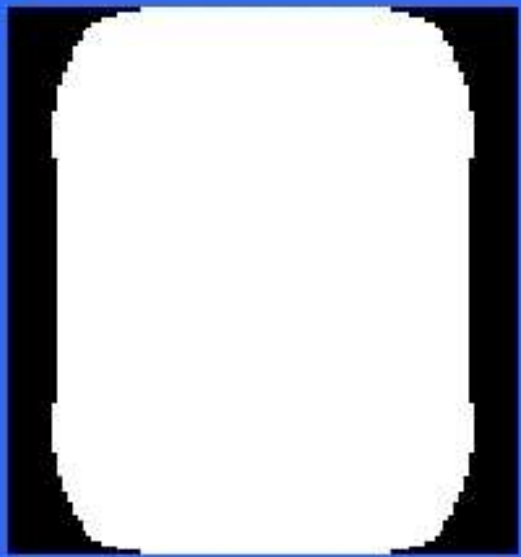

27

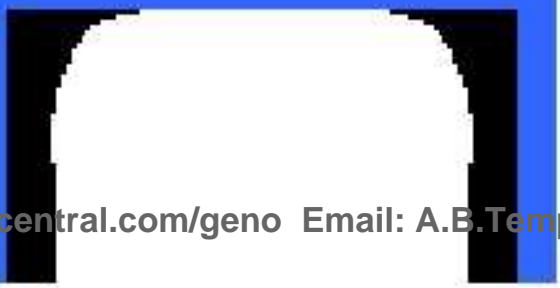

pleme

28 
Page 35 offisfineering Optimization

1
2
3
4
5

1

2

3

4

5

6

7

8

9

10

11

12

13

14

15

16

17

18

19

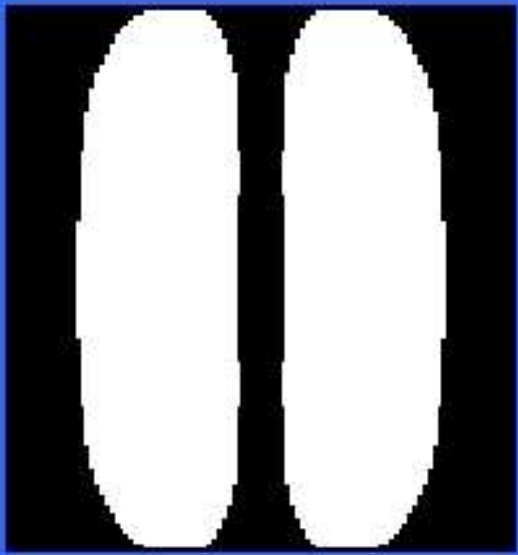

20

21

22

23

24

25

u 26

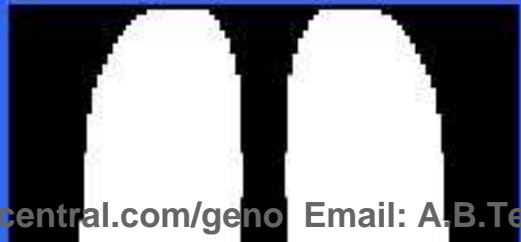

pleme

27

28 
Enaineering_Optimiza

1
2
3
4
5

6

7

8

9

10

11

12

13

14

15

16

17

18

19

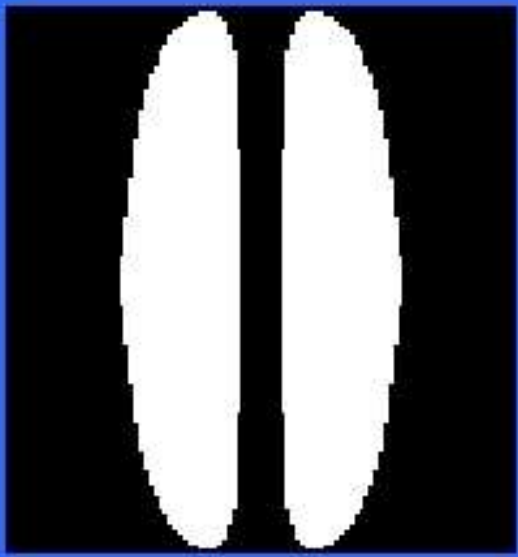

20

21

22

23

24

25

14. 26

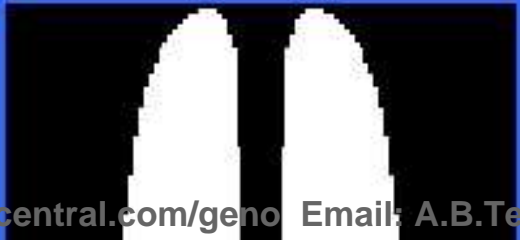

pleme

27

28 


\section{Page 37 offrimineering Optimization}

1
2
3
4
5

6

7

8

9

10

11

12

13

14

15

16

17

18

19

20

21

22

23

24

25

Ius6ri :entral.com/geno Email: A.B.Te

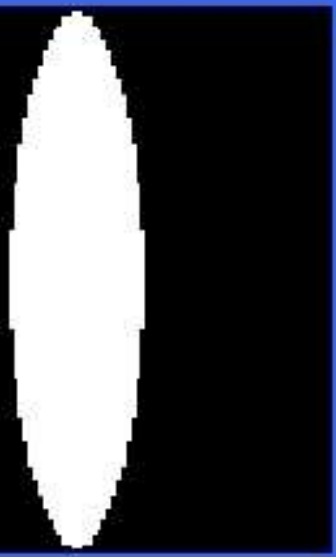

28 
Tao=0

\section{Engineering OptimizatPage 38 of 55}

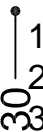

4
5

6

7

8

Tai=1

9

10

11

$n$
13

14

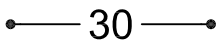

$\mathrm{Tao}=0$

$\Omega$

Email: A.B.Templemaı . 


1
2
3
4
5
6
7
8
9

10

11

12

13

14

15

16

17

18

19

20

21

22

23

24

25

http:/mc.manuscriptcentral.com/geno Email: A.B.Templeman@liverpoo|.ac.ul 27

28 
Engineering-Optimizalliage 40 of 55

1
2
3
4
5
6
7
8
9

10

11

12

13

14

15

16

17

18

19

20

21

22

23

24

25

u 26
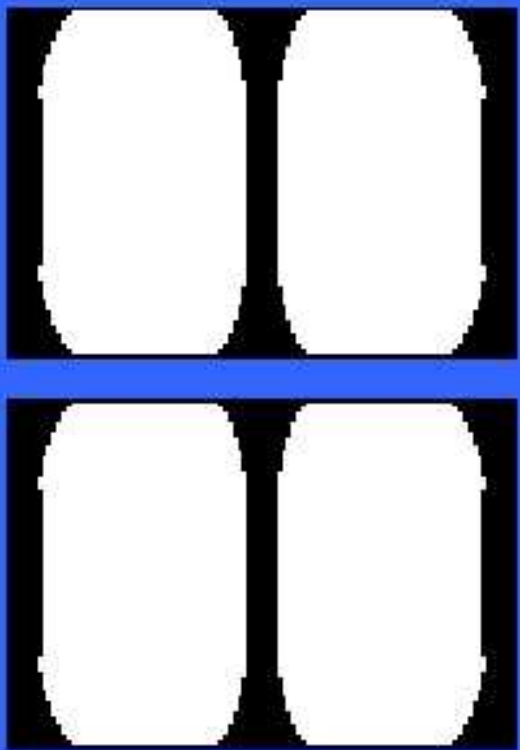

27

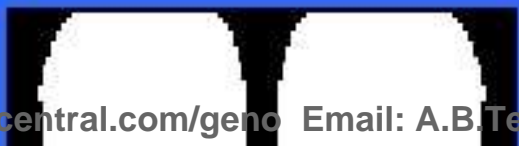

pleme

28 
Page 41 dfistgineerina Optimization

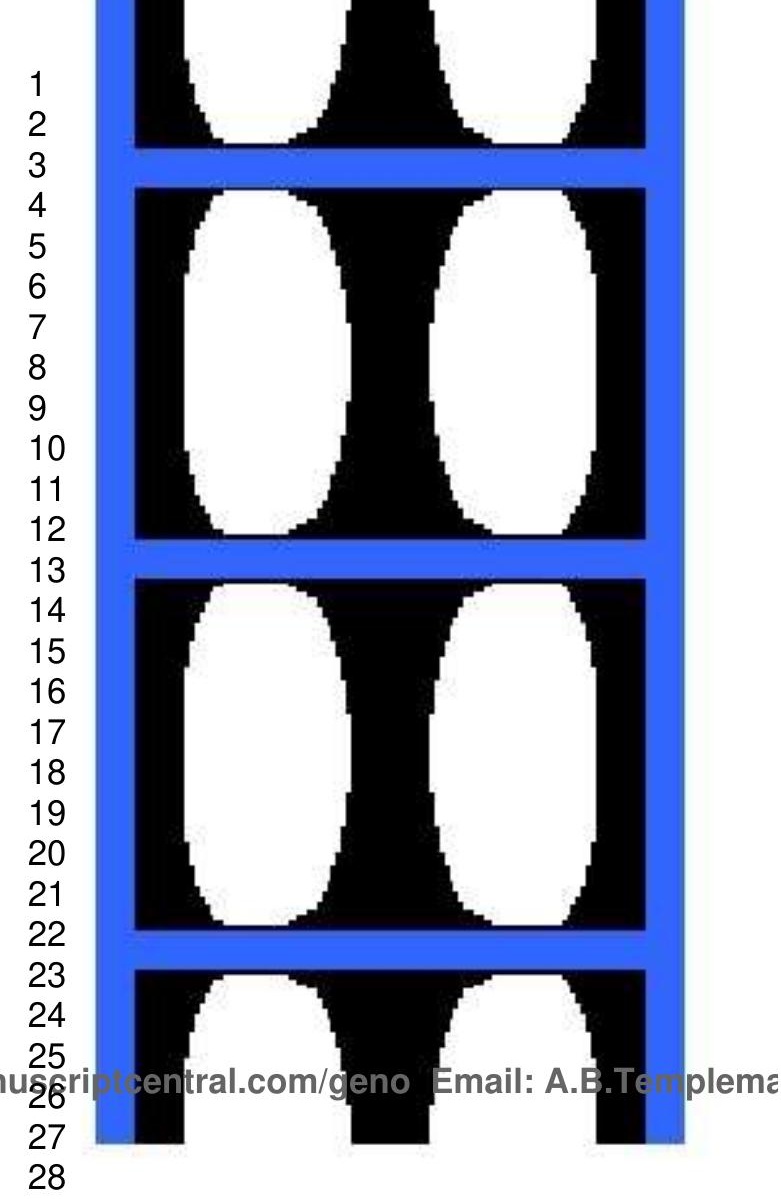


Engineerino_Ontimizalfiame_42 of 55

3

4

5

6

7

8

9

10

11

12

13

14

15

16

17

18

19

20

21

22

23

24

25

IU⿺ 26

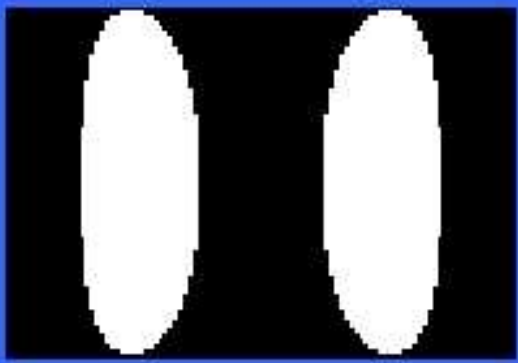

27

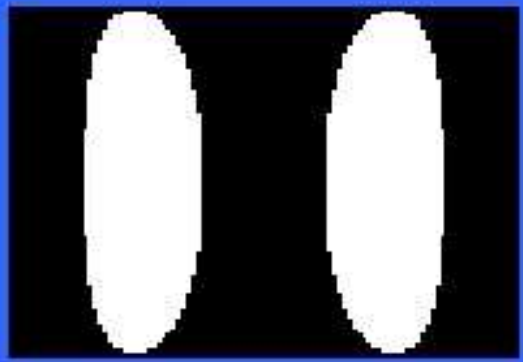

28

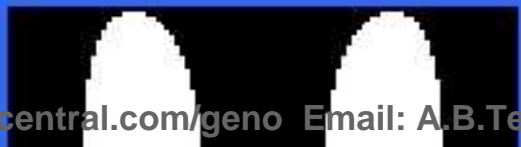

pleme 
Page 43 offifineerina Ontimization

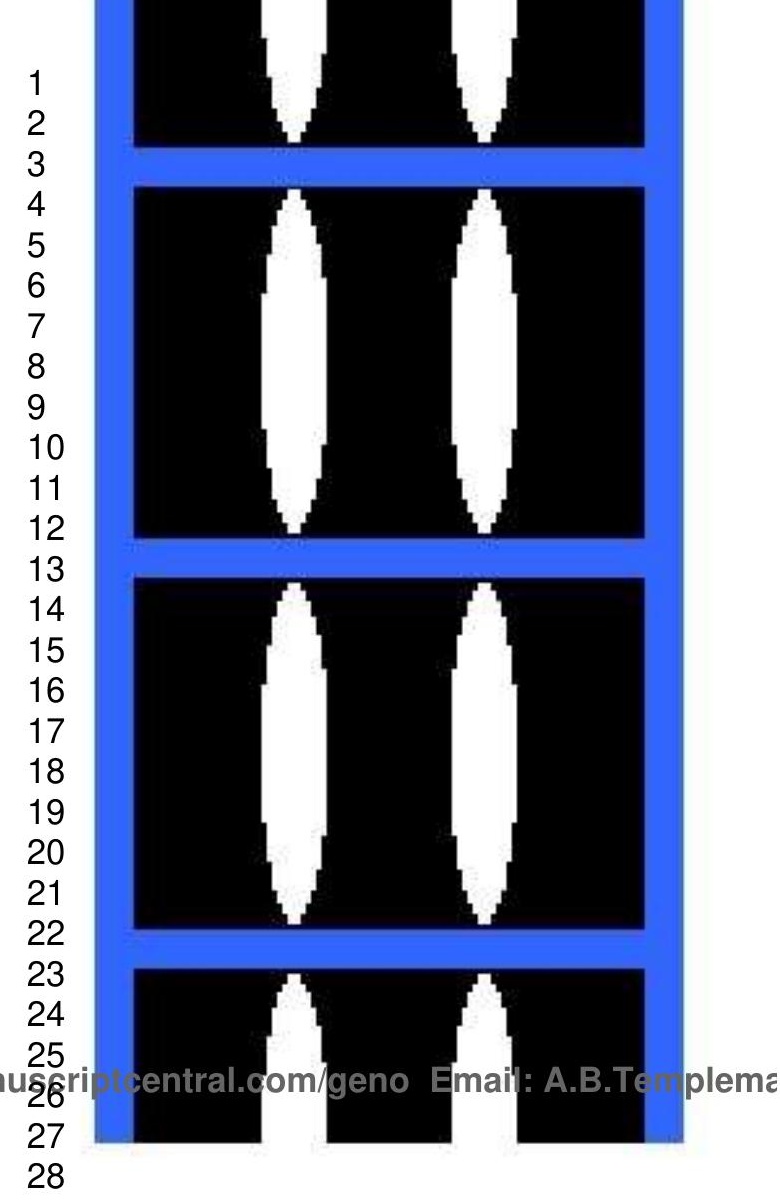


Engineering Qptimizeatgen 44 of 55

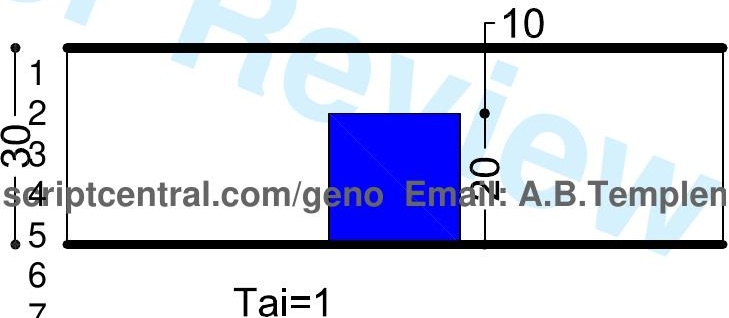


Page 45 of 55

Engineering Optimization

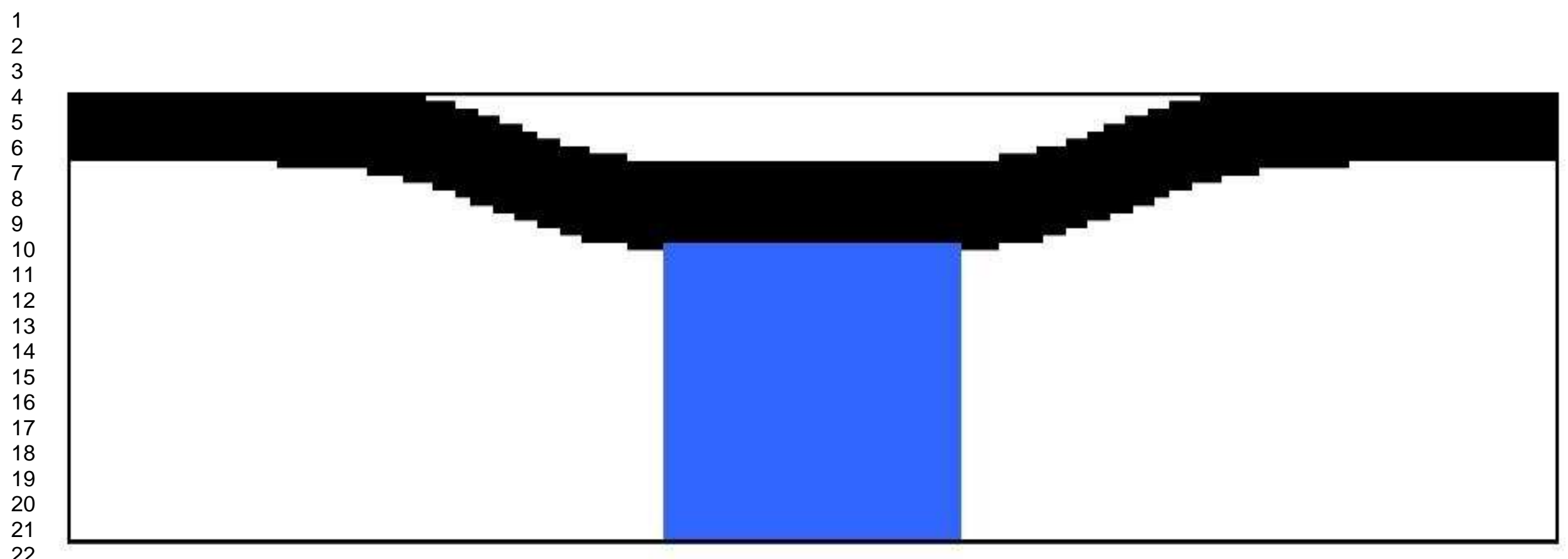

23

24

25

URL: http:/mc.manuscriptcentral.com/geno Email: A.B.Templeman@liverpool.ac.uk

26

27 
Tao=0

\section{Engineering OptimizatPerge 46 of 55}

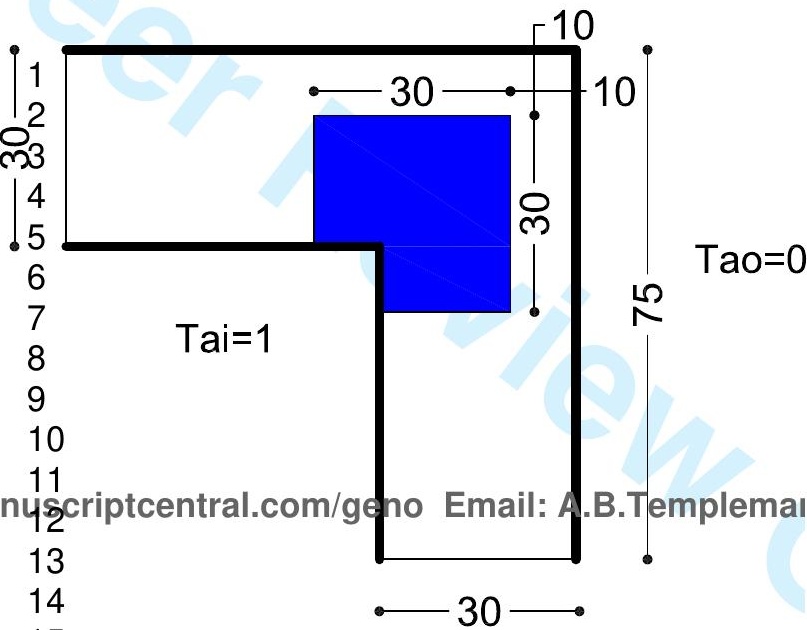




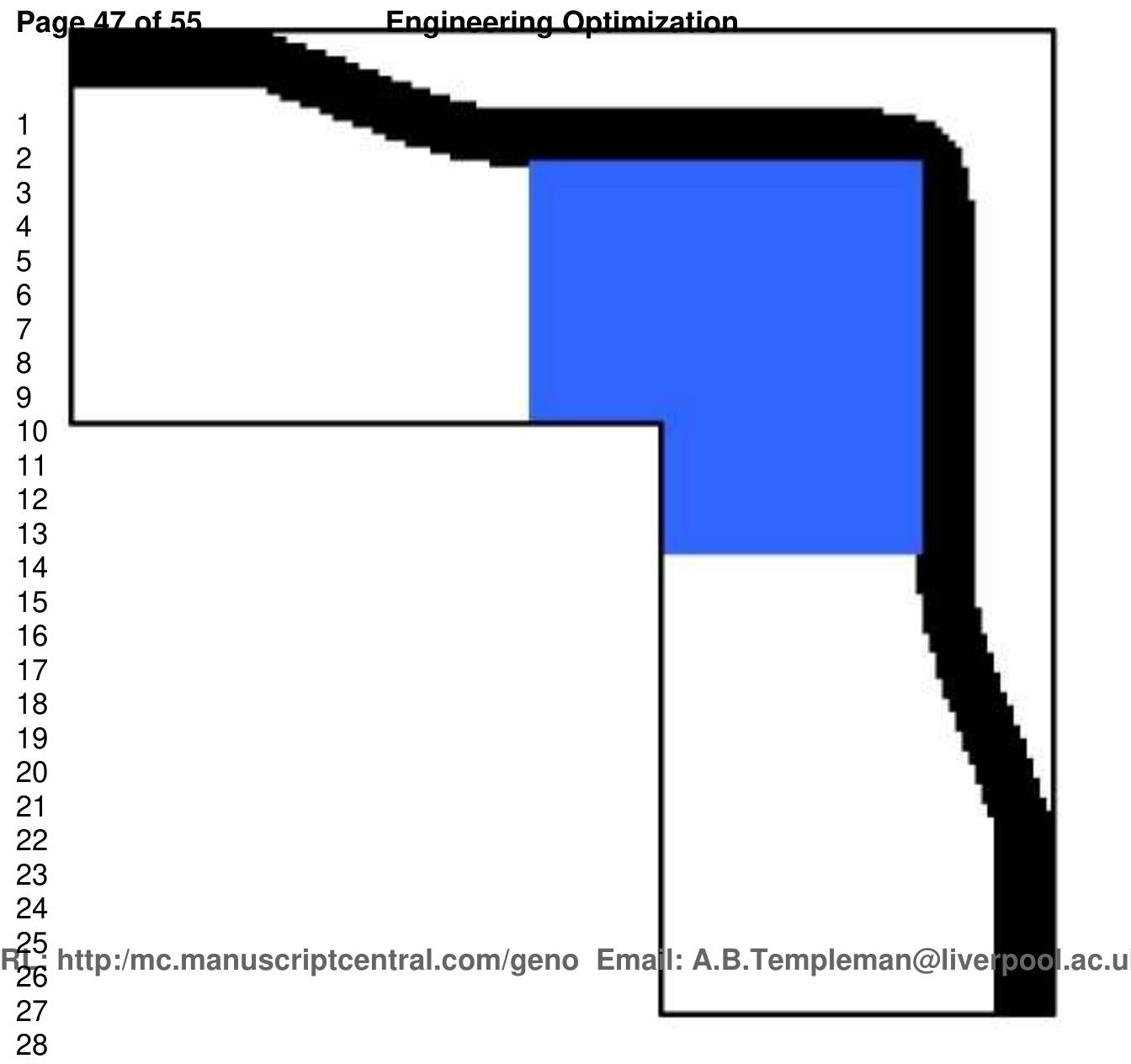




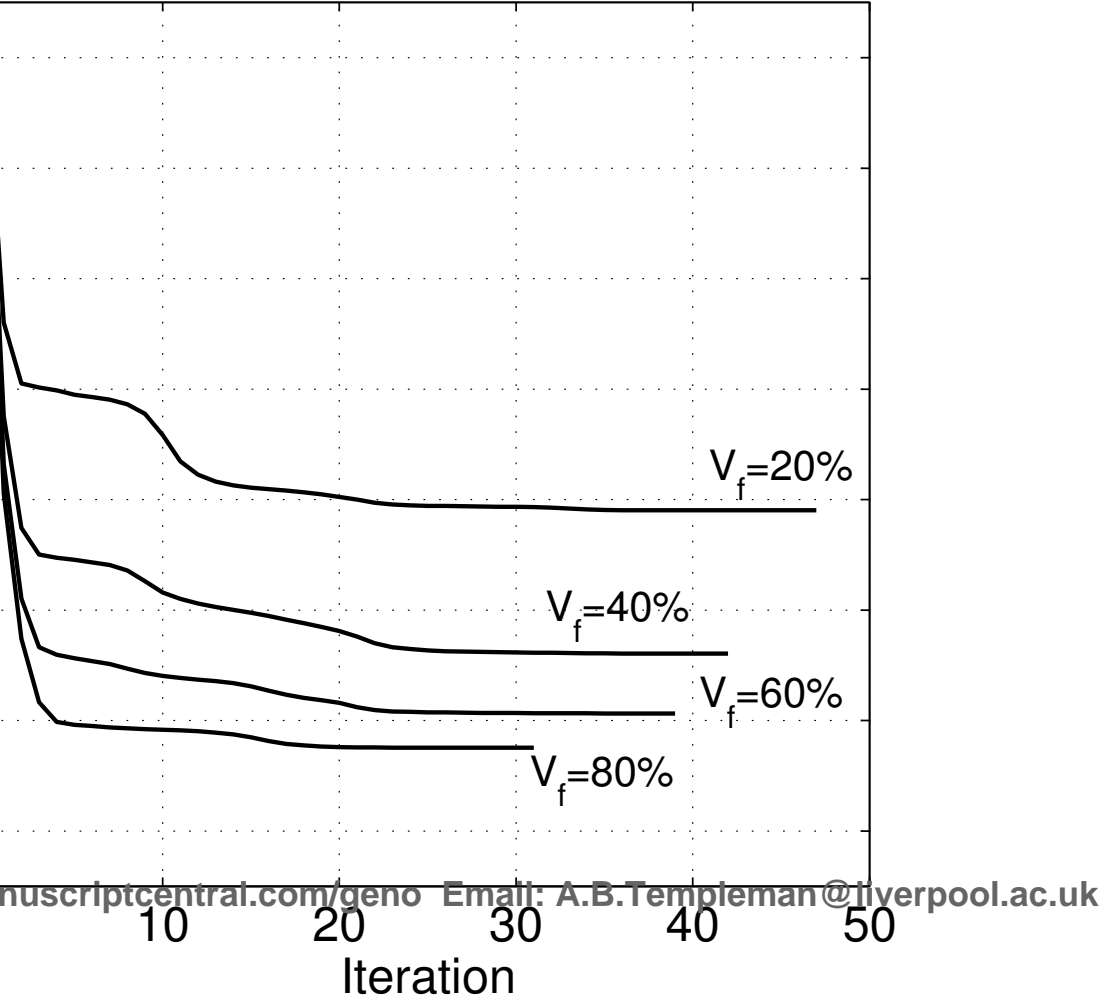



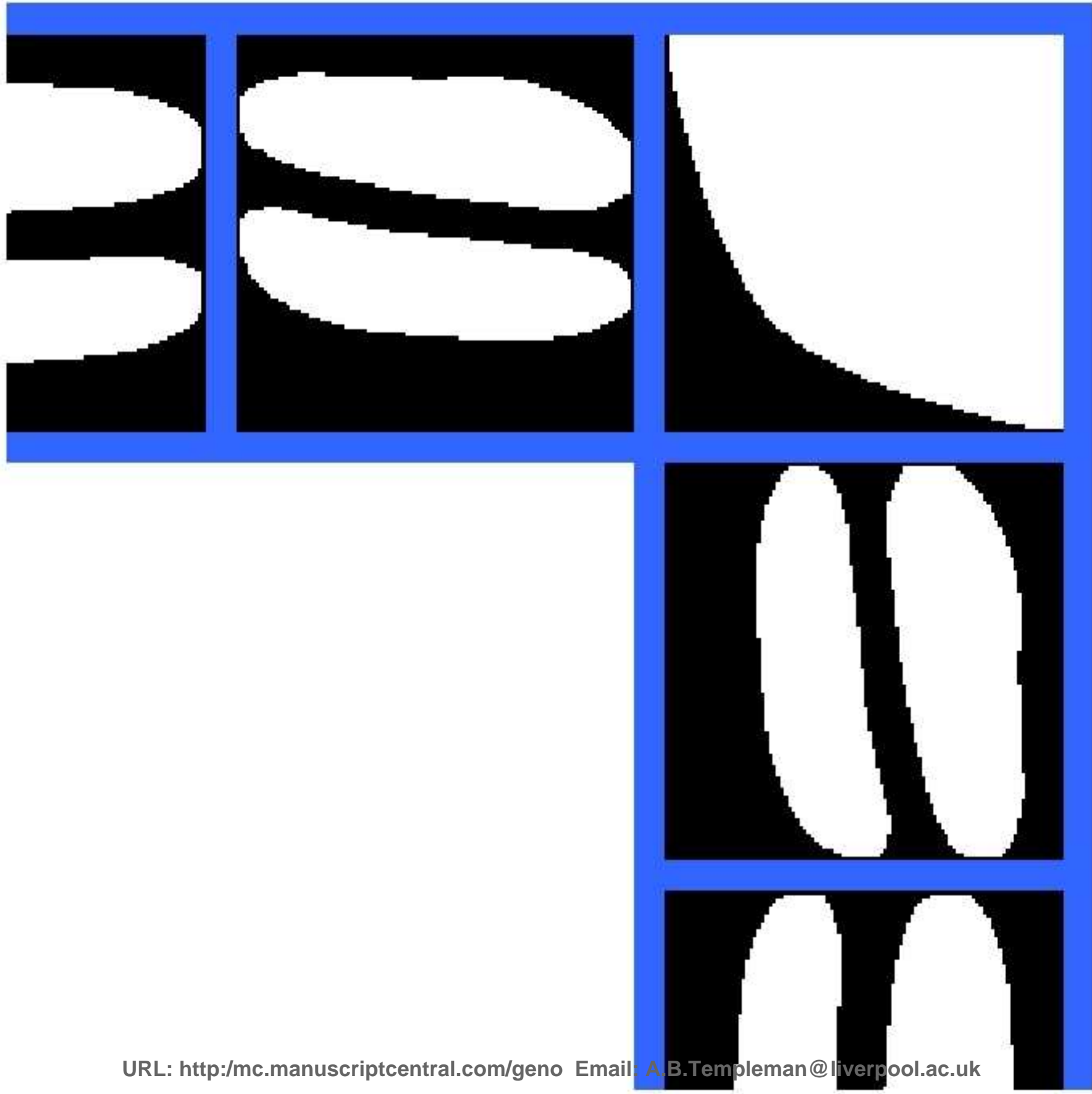


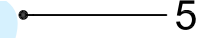

5

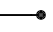

$5 \longrightarrow$

Eregfreering Optimization Page 50 б自5 0

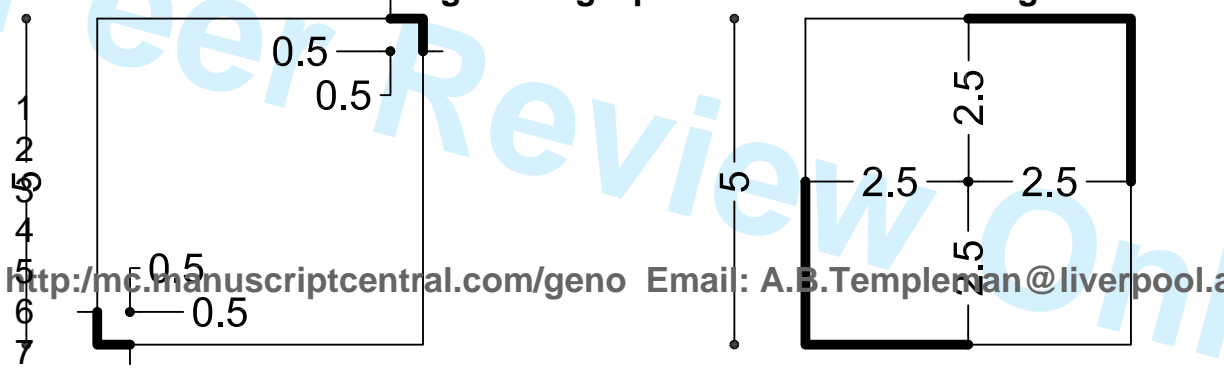

$\mathrm{T} 8 \mathrm{i}=1$

Tai $=1$ 
Tao=0

\section{Engineering Optimiz fratge 52 of 55}

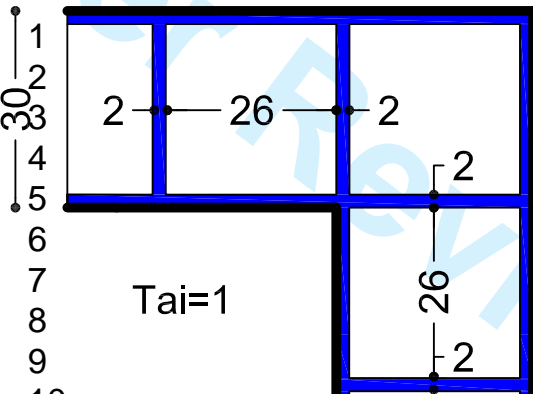

$\left.\right|_{T a O=0}$

usq9iptcentral.com/geno Email A.B.Templem:

12

13

30 


\section{Page 53 of 55}

Convergence opMaxproblems

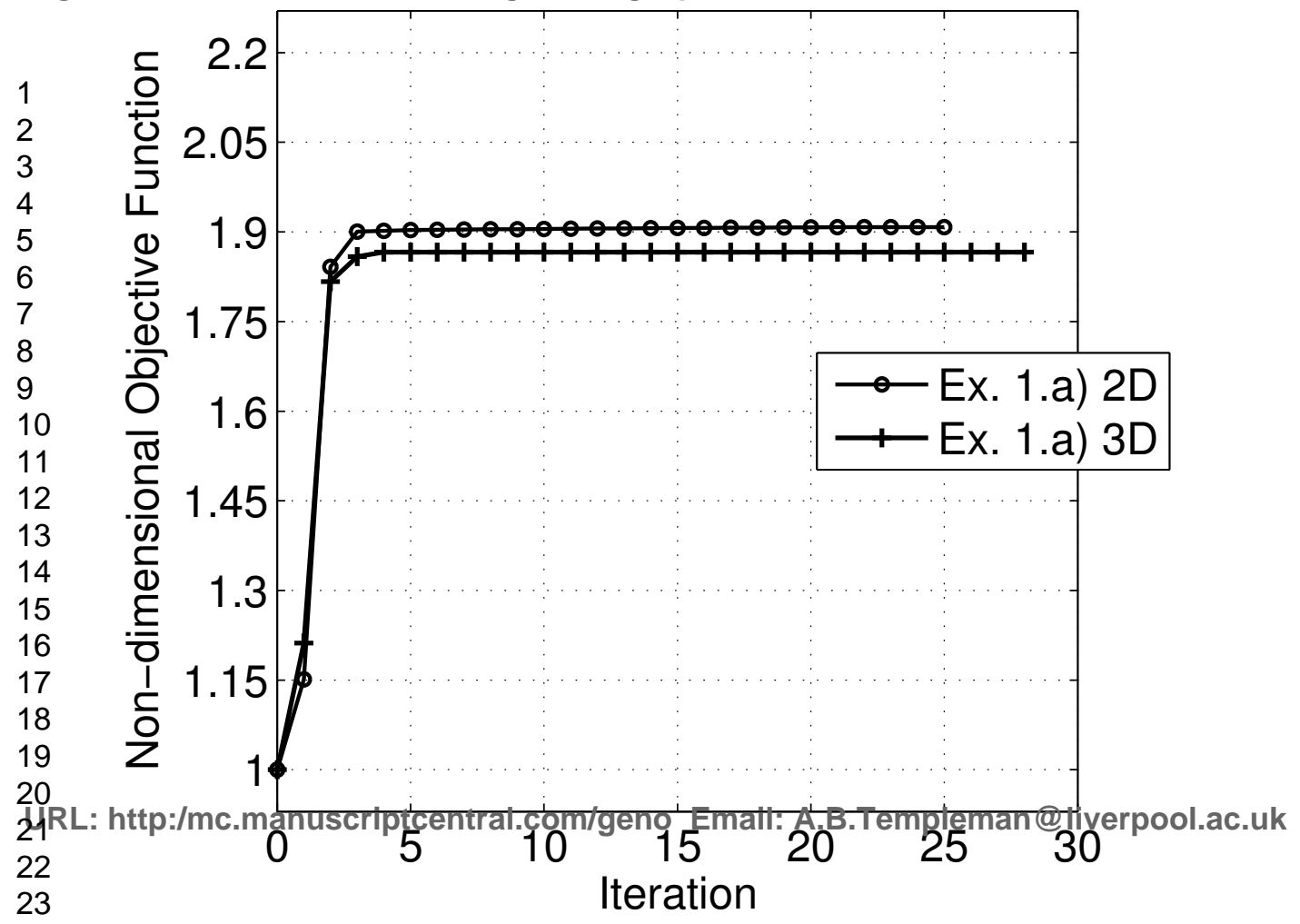


Convergence -Min problems

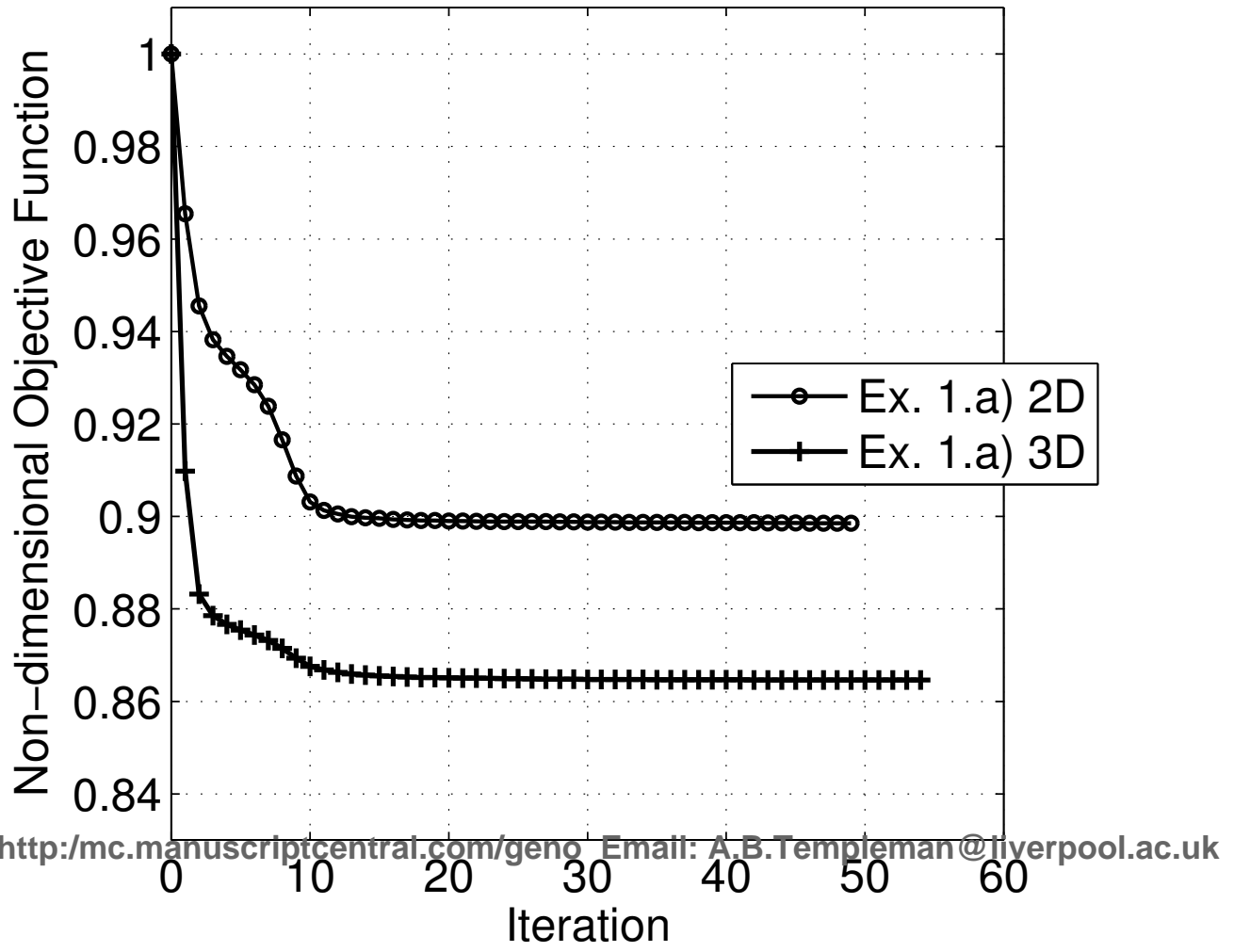




\section{Page 55 of 55 Thermal performance}

1

2

3

4

5

6

7

8

9

10

11

12

13

14

15

16

17

18

19

20

21RL: http:/mc.manuggriptcentral.cq0/geno EmaibO.B.Templem80@liverpool.ac.uk 22

23

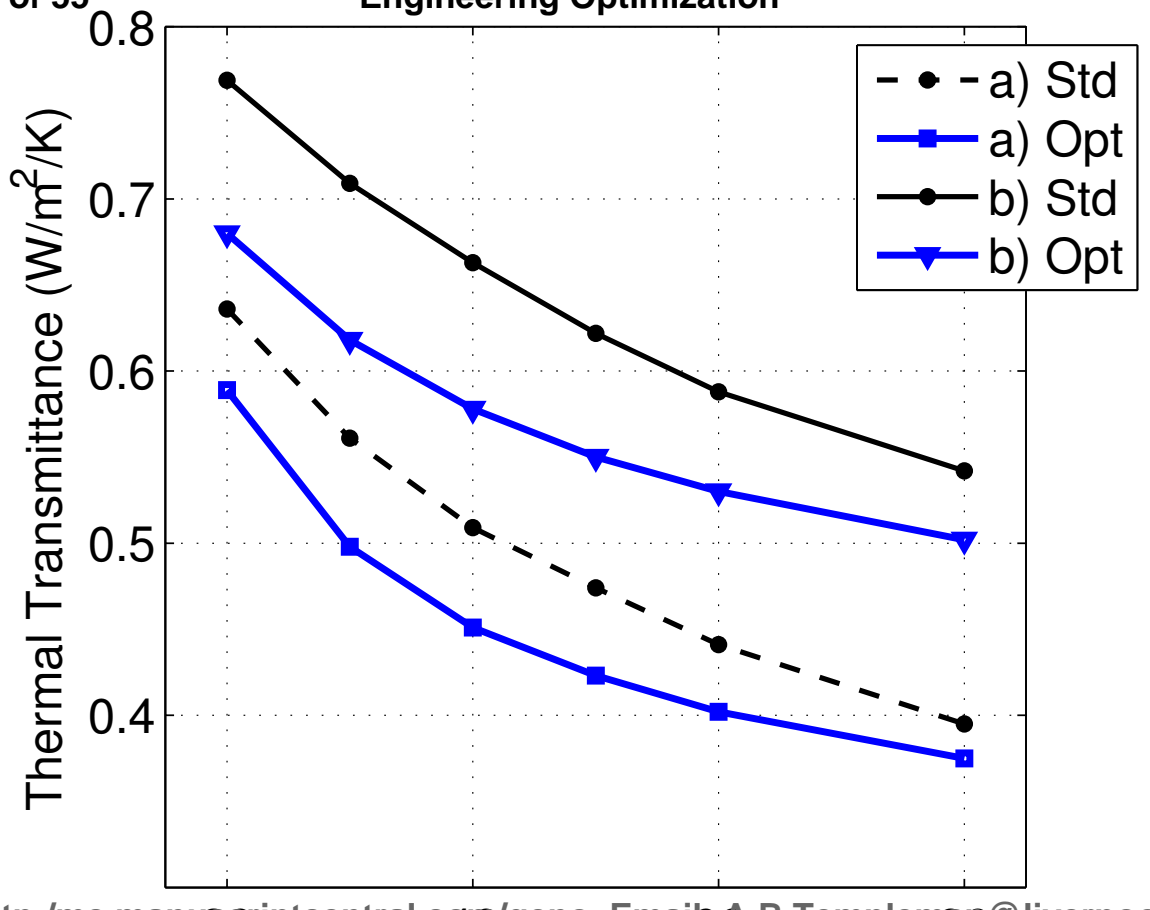

$V_{f}(\%)$ 